\title{
Identification and localization of layers in the ionosphere using the eikonal and amplitude of radio occultation signals
}

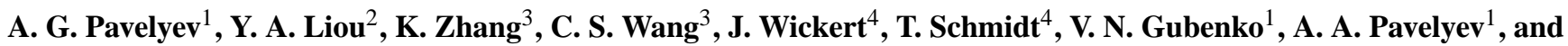 \\ Y. Kuleshov ${ }^{3}$ \\ ${ }^{1}$ Kotelnikov Institute of Radio Engineering and Electronics of the Russian Academy of Sciences, Fryazino, \\ Moscow region, Russia \\ ${ }^{2}$ Center for Space and Remote Sensing Research, National Central University, Chung-Li, 320, Taiwan \\ ${ }^{3}$ SPACE Research Centre, RMIT University, G.P.O. Box 2476V, Melbourne, Victoria 3001, Australia \\ ${ }^{4}$ GeoForschungsZentrum Potsdam (GFZ-Potsdam), Telegrafenberg, 14473 Potsdam, Germany
}

Correspondence to: A. G. Pavelyev (alxndr38@mail.ru)

Received: 19 January 2011 - Published in Atmos. Meas. Tech. Discuss.: 1 March 2011

Revised: 4 November 2011 - Accepted: 23 November 2011 - Published: 4 January 2012

\begin{abstract}
By using the CHAllenge Minisatellite Payload (CHAMP) radio occultation (RO) data, a description of different types of the ionospheric impacts on the RO signals at the altitudes $30-90 \mathrm{~km}$ of the RO ray perigee is given and compared with the results of measurements obtained earlier in the satellite-to-Earth communication link at frequency $1.5415 \mathrm{GHz}$. An analytical model is introduced for describing propagation of radio waves in a stratified medium consisting of sectors with spherically symmetric refractivity distribution. This model gives analytical expressions for the phase, bending angle, and refractive attenuation of radio waves and is applied to the analysis of radio wave propagation phenomena along an extended path including the atmosphere and two parts of the ionosphere. The model explains significant amplitude and phase variations at altitudes 30 $90 \mathrm{~km}$ of the RO ray perigee and attributes them to inclined ionospheric layers. Based on this analytical model, an innovative technique is introduced to locate layers in the atmosphere and ionosphere. A necessary and sufficient criterion is obtained for a layer to be located at the RO ray perigee. This criterion gives both qualitative and quantitative estimation of the displacement of an ionospheric and/or atmospheric layer from the RO ray perigee. This is important, in particular, for determining the location of wind shears and directions of the internal wave propagation in the lower ionosphere, and, possibly, in the atmosphere.
\end{abstract}

\section{Introduction}

High-stability radio signals transmitted at two GPS (Global Positioning navigational System) frequencies $f_{1}=$ $1575.42 \mathrm{MHz}$ and $f_{2}=1227.60 \mathrm{MHz}$ from a GPS satellite and received at a GPS receiver aboard a low Earth orbit (LEO) small/micro satellite have been used in radio occultation (RO) investigations of the atmosphere (Melbourne et al., 1994; Ware et al., 1996; Kursinki et al., 1997; Hajj and Romans, 1998; Steiner et al., 1999; Wickert et al., 2001, 2004; Gorbunov et al., 2002, 2010; Yakovlev, 2003; Jensen et al., 2003, Melbourne, 2004; Gorbunov and Lauritsen, 2004; Gorbunov and Kirchengast, 2005; Pavelyev et al., 2002, 2004, 2009; Liou and Pavelyev, 2006; Liou et al., 2002, $2003,2005,2007,2010)$. When applied to ionospheric investigations, the RO method may be considered as a global tool and can be compared with the global ground- and spacebased radio tomography (e.g. Jakowski et al., 2004; Kunitsyn and Tereschenko, 2003, and references therein). RO measurements in the atmosphere can be impacted by ionospheric contributions because the RO signal propagates through two different parts of the ionosphere. Usually, the ionospheric influence can be described as a relatively slow change in the excess phase path without noticeable variations in the amplitude of the RO signal. This change can be excluded by different methods of ionospheric correction (Melbourne et al., 1994; Vorob'ev and Krasilnikova 1994; Gorbunov, 2002). Disturbed ionosphere significantly changes not only the phase but also the amplitude of the RO signals. The 


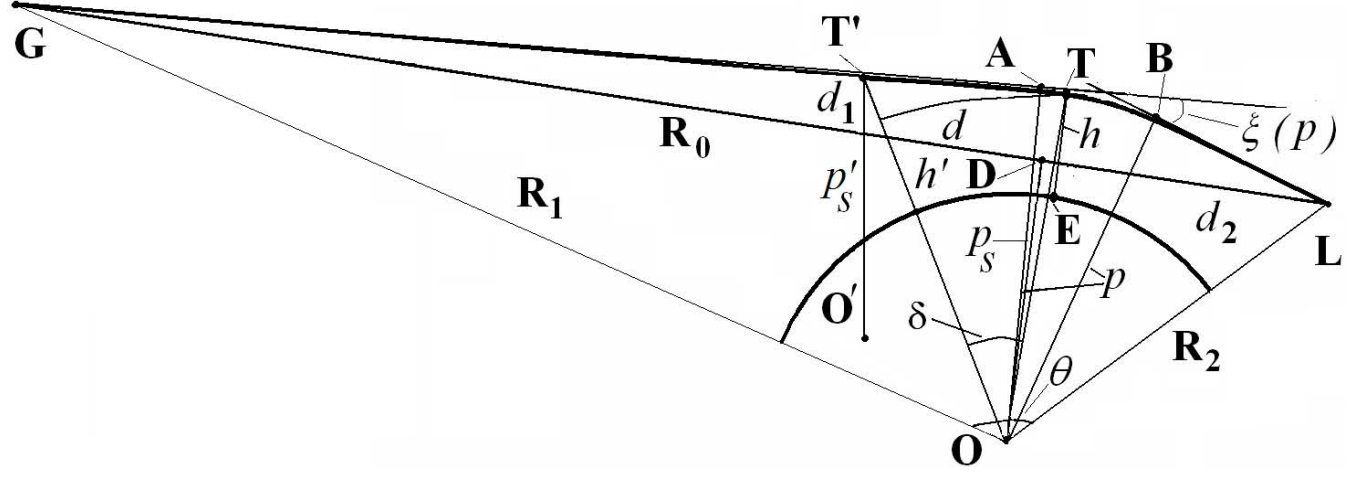

Fig. 1. Geometry of radio ray path in RO experiment.

phenomenon of strong amplitude and phase frequency dependent variations in the RO signals is often observed within the altitudes of the RO ray perigee $h$ between 30 and $90 \mathrm{~km}$ above the main part of the neutral atmosphere and below the E-layer of the ionosphere. This effect is also interesting for investigating propagation of radio waves and communication in the satellite-to-satellite links. The amplitude of the RO signal offers new possibilities for studying the ionosphere (Vorob'ev et al., 1997; Sokolovskiy, 2000; Sokolovskiy et al., 2002; Igarashi et al., 2000, 2001; Gorbunov et al., 2002; Pavelyev et al., 2002, 2004, 2007, 2008a, b, 2009, 2010a; Liou et al., 2002, 2003, 2005, 2007; Liou and Pavelyev, 2006). Igarashi et al. (2000, 2001); Pavelyev et al. (2002), indicated high sensitivity of the RO amplitude (as compared with the eikonal) on features of plasma layers in the E-region of the ionosphere. Comparison of the eikonal and amplitude variations in the Microlab-1 RO data and estimation of sporadic E-layers parameters made by Vorob'ev et al. (1997). Gorbunov (2002), and Sokolovskiy et al. (2002) proposed a back-propagation radio-holographic method for investigation of ionospheric layers and irregularities by using RO data.

The goals of this paper are (i) to describe different kinds of ionospheric influence on the GPS RO signals within the altitudes of $h$ between 30 and $90 \mathrm{~km}$, and to compare them with the noisy (C-type) and regular (S-type) scintillations detected earlier by Karasawa et al. (1985) in the satellite-toEarth communication link; (ii) to present an analytical model for the refractive attenuation and excess phase path (eikonal) of electromagnetic waves in locally spherical symmetric media; and (iii) to identify the contributions and to measure parameters of the inclined plasma layers by analyzing the CHAMP RO experimental data. The paper is structured as follows. In Sect. 2 the description of different kinds of the ionospheric impact on the CHAMP RO signal is presented. Section 3 introduces an analytical model of wave propagation through the ionosphere and atmosphere of the Earth. In Sect. 4 an example of ionospheric layers identification and the electron density retrieval is considered. Conclusions are given in Sect. 5.

\section{Types of ionospheric influence on the CHAMP RO signal}

During a RO event, the radio ray linking a LEO GPS receiver to a GPS satellite transmitter (marked by points L and $\mathrm{G}$ in Fig. 1, respectively) immerses gradually into the ionosphere and atmosphere. The LEO receiver measures the GPS dual-frequency phase (eikonal) delays and amplitudes of the RO signal for the subsequent determination of the vertical profiles of the physical parameters of the atmosphere (including ionosphere). In the case of single path propagation, the RO inversion technique is based on two implicit assumptions: (i) the tangent point $\mathrm{T}$, where the refractivity gradient is perpendicular to the RO ray, is located in the RO ray perigee (Fig. 1); and (ii) there is only one tangent point on the RO ray trajectory GTL. Under these assumptions, a relatively small area with a center at point $\mathrm{T}$ makes the main contribution to the amplitude and phase variations of the RO signals (Igarashi et al., 2000, 2001). In this case, the amplitude and phase variations of the RO signals are functions of the ray perigee height $h$ (Fig. 1) and the satellites' positions and velocities. Previously, the RO technology has been mainly based on analyzing the phase of electromagnetic wave transmitted through the ionosphere and atmosphere (Ware et al., 1996). Since then, different radioholography approaches using the complex field have been introduced to study the atmosphere under the assumption of spherical symmetry (e.g. Liou et al., 2010; Gorbunov et al., 2010, and references therein).

In general, the assumption of spherical symmetry in the ionosphere cannot be directly implemented to locate sporadic ionospheric disturbances (Wickert et al., 2004; Liou et al., 2005). Gorbunov et al. (2002) and Sokolovskiy et al. (2002) applied the Back Propagation (BP) radioholographic method to locate ionospheric disturbances in the E- and F-layers of the ionosphere. Wickert et al. (2004) indicated a difficulty in resolving the uncertainty in the inclined plasma layer location between the parts TL and GT of the ray trajectory GTL (Fig. 1). 

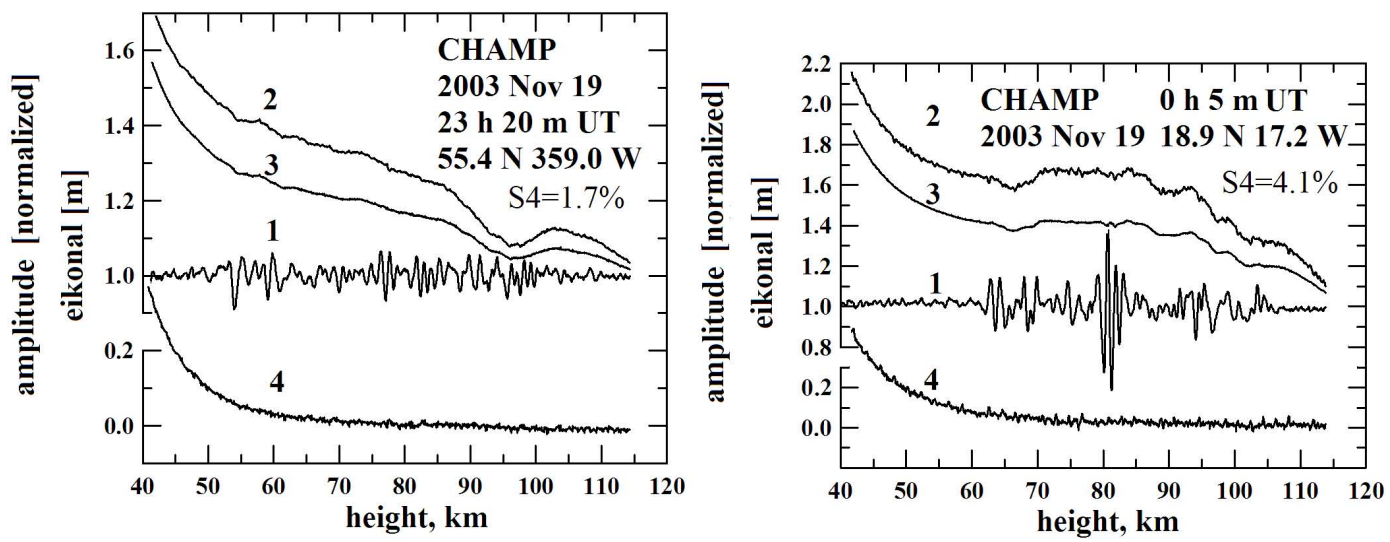

Fig. 2. Amplitude and excess phase measurements of the CHAMP RO signals in both quiet (left) and slightly disturbed (right) ionosphere (events no. 0211 and 0001, 19 November 2003). Local time of the event and the coordinates of the RO ray perigee $T$ are shown in the graphs. The $S_{4}$ index value is shown in the lower line of the insert. The amplitude curve 1 has low variations with index $S_{4}=1.7 \%$ (left) and $S_{4}=4.1 \%$ (right). The phase path (eikonal) excesses $\Phi_{1}(t)$ and $\Phi_{2}(t)$ at $f_{1}$, and $f_{2}$ are shown by curves 2 and 3 , respectively. Curve 4 corresponds to the combined eikonal $\Phi(t)$.

Amplitude data can be used to obtain the vertical gradients of the electron density in the ionosphere and refractivity in the atmosphere independently (Pavelyev et al., 2002; Liou et al., 2002; Sokolovskiy, 2000; Sokolovskiy et al., 2002). High sensitivity of the amplitude variations on ionospheric plasma layers can be used to describe different kinds of ionospheric influence on the RO signals in the trans-ionospheric communication links.

The RO experiment aboard CHAMP was activated on 11 February 2001 (Wickert et al., 2001). Occultation measurements of the ionosphere and neutral atmosphere were autonomously scheduled by the Black-Jack receiver instrument. Carrier excess phase paths (eikonals) at two frequencies $f_{1}, f_{2}$, and signal-to-noise ratio (SNR) at frequency $f_{1}$ were recorded at a sampling rate of $50 \mathrm{~Hz}$. In the case of CHAMP RO experiments, quiet ionospheric conditions have come to light in the form of small values of the $S_{4}$ index of the amplitude scintillations averaged over the altitude interval $h$ from 40 to $90 \mathrm{~km}$ :

$S_{4}=\left[<(I(t)-<I>)^{2}>/<I>^{2}\right]^{1 / 2}$

where $I$ and $<I>$ are the current and averaged intensities of the RO signals corresponding to propagation in the medium. An example of quiet and moderately disturbed ionospheric conditions observed during the CHAMP RO experiments is shown in Fig. 2 (left and right panels, respectively). The amplitude curve 1 has low variations with index $S_{4}=1.7 \%$ (left) and $S_{4}=4.1 \%$ (right). The phase path (eikonal) excesses $\Phi_{1}(t)$ and $\Phi_{2}(t)$ at $f_{1}$, and $f_{2}$ are shown by curves 2 and 3, respectively. Curve 4 corresponds to the combined eikonal $\Phi(t)$. The combined eikonal $\Phi(t)$ (curve 4 ) has been evaluated from the following ionospheric correction formula (Melbourne et al., 1994):

$\Phi(t)=\left[f_{1}^{2} \Phi_{1}(t)-f_{2}^{2} \Phi_{2}(t)\right] /\left(f_{1}^{2}-f_{2}^{2}\right)$
Examples of significant variations in the phase and amplitude of the GPS RO signals are given below for the ray perigee altitudes $40-90 \mathrm{~km}$. These examples support the suggestion that there exist two types of ionospheric impact on the RO signals: noisy and regular ones which correspond to $\mathrm{C}$ - and S-types of ionospheric influence on the amplitude of radio waves propagating in the INMARSAT-Earth communication link at frequency $1541 \mathrm{MHz}$ (Karasawa et al., 1985). When the regular variations of the RO signal are caused by an inclined plasma layer, the displacement $d$, correction to the height of the ionospheric layer $\Delta h$ and its inclination $\delta$ with respect to the local horizontal direction (Fig. 1), are connected (Wickert et al., 2004) via equations:

$\delta=d / r_{\mathrm{e}}, \Delta h=0.5 d \delta, d=\left(2 \Delta h r_{\mathrm{e}}\right)^{1 / 2}$

where $r_{\mathrm{e}}$ is the distance TO (Fig. 1).

A RO event with the isolated quasi-regular influence of sporadic E-layers is shown in Fig. 2 (right panel). The fine regular structures are seen in the altitude interval $h$ of 65 $90 \mathrm{~km}$. These vertical phase and amplitude distributions can correspond to a sporadic E-layer, which is usually located at a height of about $90-130 \mathrm{~km}$ in the ionosphere. Therefore, the height of a sporadic layer evaluated as $h$ is underestimated and should be corrected with accounting for the displacement of the centre of spherical symmetry as it follows from the analytical model introduced in Sect. 3. An example of the event with high quasi-regular variations in the amplitude and eikonal of the CHAMP RO signal with the magnitude of the index $S_{4}=17 \%$ is shown in Fig. 3. The eikonals at two frequencies vary in the interval $1.5 \leq \Phi_{1,2} \leq 10.3 \mathrm{~m}$. The variations at $48-58 \mathrm{~km}$ altitudes can be attributed to layers in the electron density distribution. Strong amplitude variations with small vertical periods of about $0.4-0.5 \mathrm{~km}$ are demonstrated in Fig. 4 (right) at the heights $98-105 \mathrm{~km}$. These 


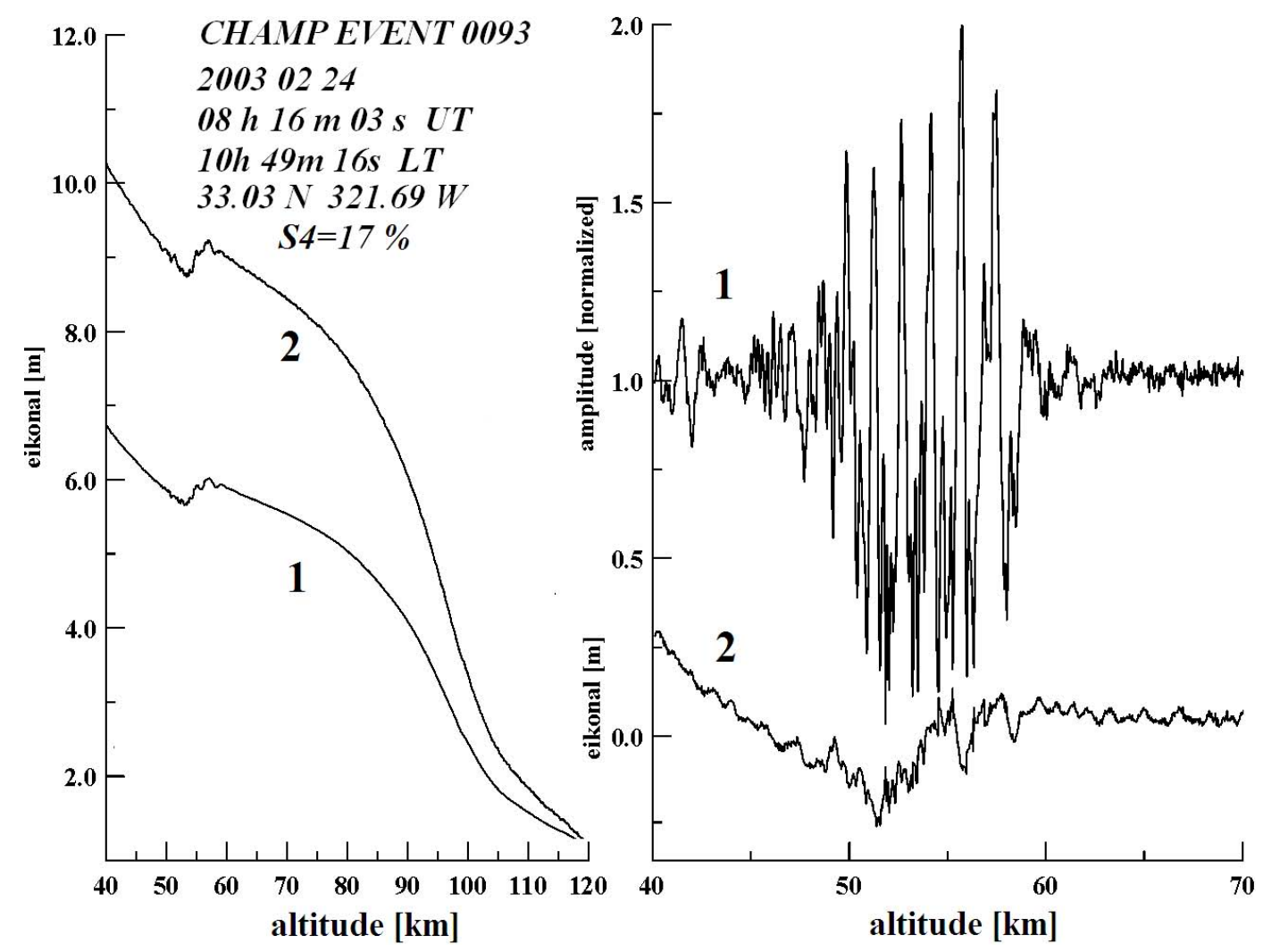

Fig. 3. Quasi-regular variations in the eikonal and amplitude values of the CHAMP RO signals for event no. 0093 (24 February 2003 ). Left: eikonal variations at frequencies $f_{1}$ and $f_{2}$ (curves 1 and 2, respectively). Right: the amplitude variations at frequency $f_{1}$ (curve 1 ) and combined eikonal $\Phi(t)$ (curve 2).

vertical periods are essentially well below the vertical size of the Fresnel zone. Therefore, these amplitude variations with short vertical periods can be considered as a consequence of the diffraction of electromagnetic waves by the sharp gradients of the electron density in a sporadic E-layer. An example of a noisy CHAMP event with large amplitude variations having small-scale vertical periods $\left(S_{4}=0.18\right)$ is shown in Fig. 5. This event can be classified as a typical case, corresponding to a noisy ionospheric contribution caused by intense ionospheric irregularities in the equatorial region in the midnight at 02:00 h Local Time (LT). The main part of the amplitude variations is limited to the interval $0.5 \leq A \leq 1.4$. These noisy variations may, possibly, correspond to bubbles moving in the disturbed region of the ionosphere.

The types of ionospheric influence (Figs. 2-5) can be compared with the results obtained earlier by Karasawa et al. (1985), who investigated the temporal characteristics of the signals from a MARISAT satellite over the Indian Ocean at an elevation angle of $17.3^{\circ}$ using long-term (13 months) measurements. Both C- and S-types of the ionospheric amplitude scintillations of radio signals are identified. The Ctype (Fig. 3, Karasawa et al., 1985) is similar to the noisy amplitude variations of the RO signal (Fig. 5). The S-type detected by Karasawa et al. (1985) consists of quasi-regular structures that can be associated with the influence of bubbles or other types of disturbances (e.g. layers) in the ionospheric plasma. Two examples of S-type variations of the relative signal level in the trans-ionospheric geostationary satellite-Earth communication link at frequency $1.5415 \mathrm{GHz}$ (Karasawa et al., 1985) are demonstrated in Fig. 6 (left and right panels). These examples can be compared with the results of simulation of the ionospheric impact on the RO signal using an analytical model of radio wave propagation in a spherically-symmetric medium (Pavelyev et al., 1996) (Fig. 7). The height of the RO ray perigee usually changes nearly uniformly with time. Therefore, the dependence on height in Fig. 7 corresponds (in some scale) to the dependence on time in Fig. 6. The width of the ionospheric layer is nearly equal to that one of the amplitude response (Fig. 7, curves 1 and 5, respectively). This indicates a possibility to determine the vertical size of an ionospheric layer as the width of the corresponding amplitude response. As follows from the data in Figs. 6 and 7, there is a good agreement between the temporal behavior of the intensity of signals in the satellite-to-Earth and satellite-to-satellite communication links. Another example of the S-type amplitude variations in the RO data is given in Fig. 8. In Fig. 8 (left panel), a comparison is given between the refractive attenuation recalculated 

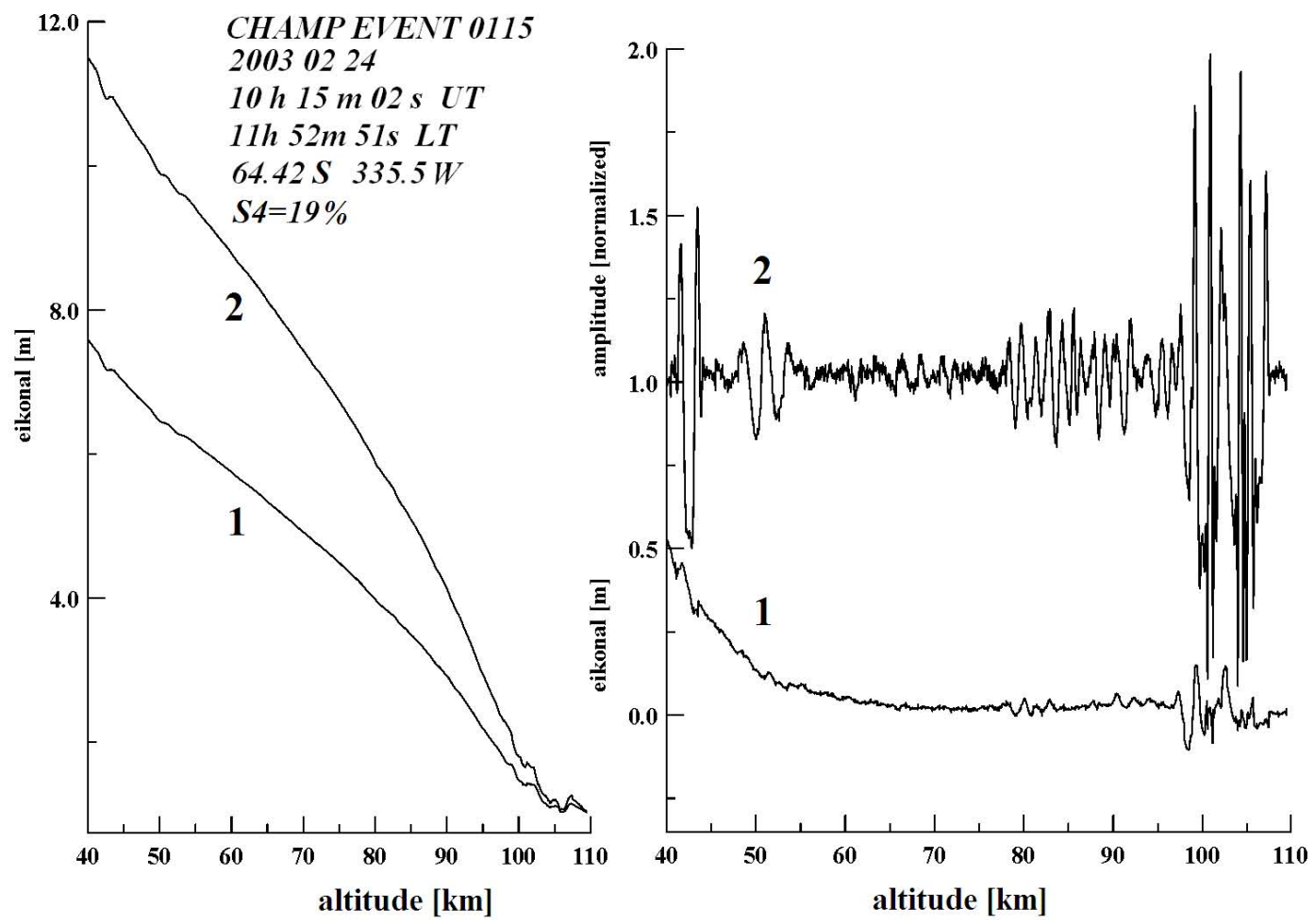

Fig. 4. Amplitude and phase values of the CHAMP RO signals due to the diffraction of electromagnetic waves in the ionosphere (event no. 0115, 24 February 2003). (Left) Eikonal variations at frequencies $f_{1}$ and $f_{2}$ (curves 1 and 2, respectively). (Right) The combined eikonal $\Phi(t)$ (curve 1) and amplitude variations at frequency $f_{1}$ (curve 2).

from the excess phase path data $X_{\mathrm{p}}$, and the refractive attenuation $X_{\mathrm{a}}$ found from the amplitude data at the first GPS frequency $f_{1}$ (curves 1 and 2). Once again one can see a good agreement between the S-type variations observed by Karasawa et al. (1985) and the temporal amplitude variations of the RO signal. As follows from Figs. 6-8, one can conclude that S-type of the amplitude scintillations observed by Karasawa et al. (1985) corresponds to the regular amplitude scintillations obtained by the analysis of the GPS RO signals. This coincidence of the types of CHAMP RO amplitude scintillations and the amplitude variations observed in the Earthbased experiments indicates that they have a common ionospheric mechanism. This conclusion is important for estimating the ionospheric interference on the conditions for communication, navigation, guidance and control of radio-links in the ionosphere.

\section{Analytical model for the excess phase path and refractive attenuation of the $\mathrm{RO}$ signals}

The effects of large-scale horizontal gradients on RO measurements have been considered by Ahmad and Tyler (1999). In this section, we describe analytical relationships for the excess phase path (eikonal), bending angle, and refractive attenuation appropriate for the trans-ionospheric satellite-to- satellite communication links. One can assume a local spherical symmetry of the electron density distributions along the ray trajectory in the entrance and exit parts of the ionosphere. These parts of the ionosphere may have different centers of spherical symmetry (e.g. point $\mathrm{O}^{\prime}$ in Fig. 1). The amplitude and phase variations of the RO signals in this case may be investigated using an analytical model appropriate for analytical ray tracing. Analytical ray tracing uses analytical presentations of the amplitude and phase (eikonal) of propagating field by use of the analytical form of refractivity distribution (e.g. Pavelyev et al., 1996). Analytical ray tracing is useful to control different regimes of the RO signal propagation (multipath, diffraction, waveguide, etc.), and is applied, in particular, to the analysis of radio wave propagating in two significantly distinct parts of the ionosphere with different distributions of electron concentration. Analytical ray tracing will be performed in a more general case for the analysis of radio communication in trans-ionospheric links (satelliteto-satellite, satellite-to-Earth). The analysis of the RO signal variations can be compared with the results obtained by the analytical model with a view to find the distribution of the electron density and to estimate the location of inclined layers in the ionosphere following the method described by Liou and Pavelyev (2006); Pavelyev et al. (2009). 

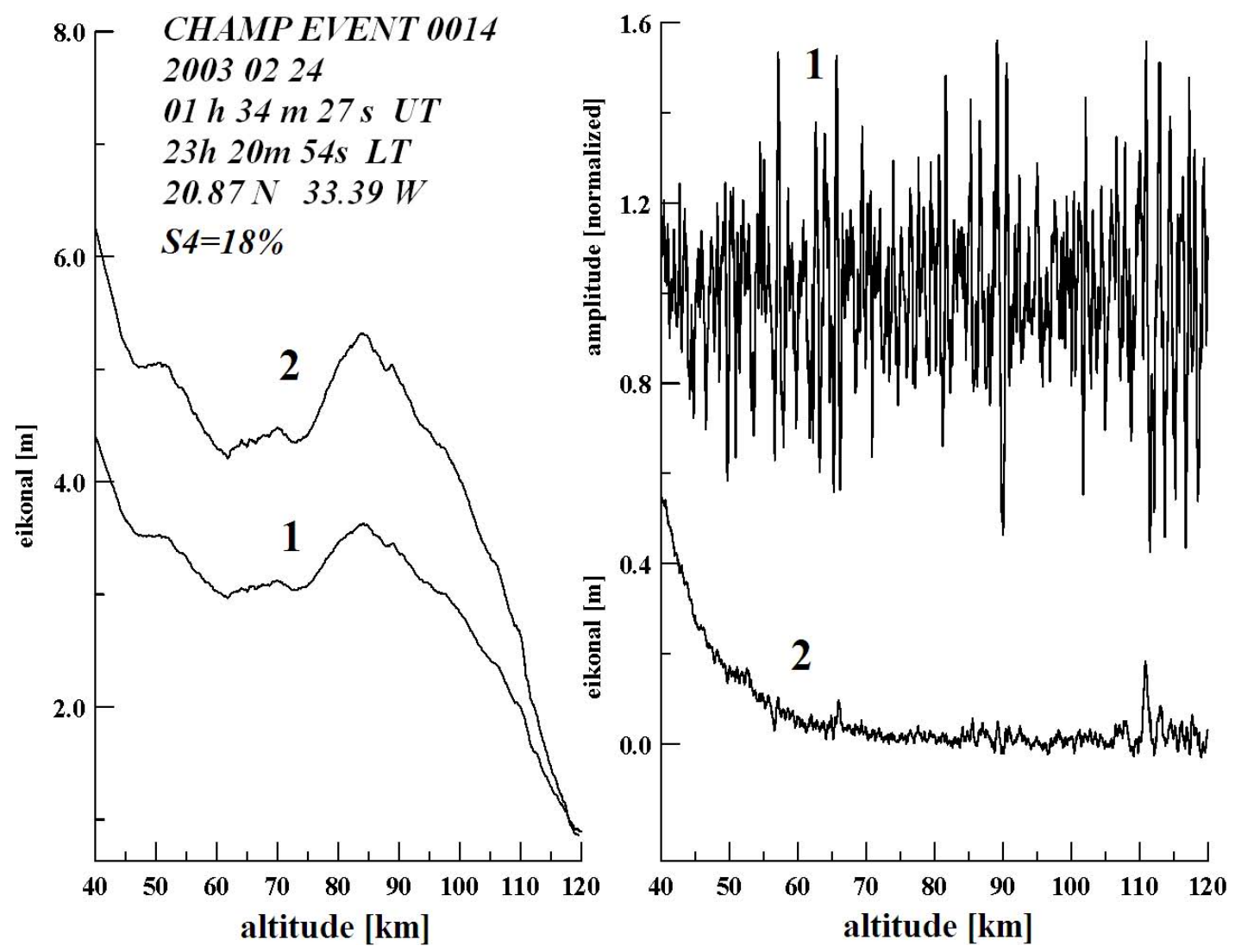

Fig. 5. Amplitude and phase values of the CHAMP RO signal for noisy events no. 0014, 24 February 2003 near the geomagnetic equator at local night. (Left) Eikonal variations at frequencies $f_{1}$ and $f_{2}$ (curves 1 and 2, respectively). (Right) Amplitude variations at frequency $f_{1}$ (curve 1) and the combined eikonal $\Phi(t)$ (curve 2).
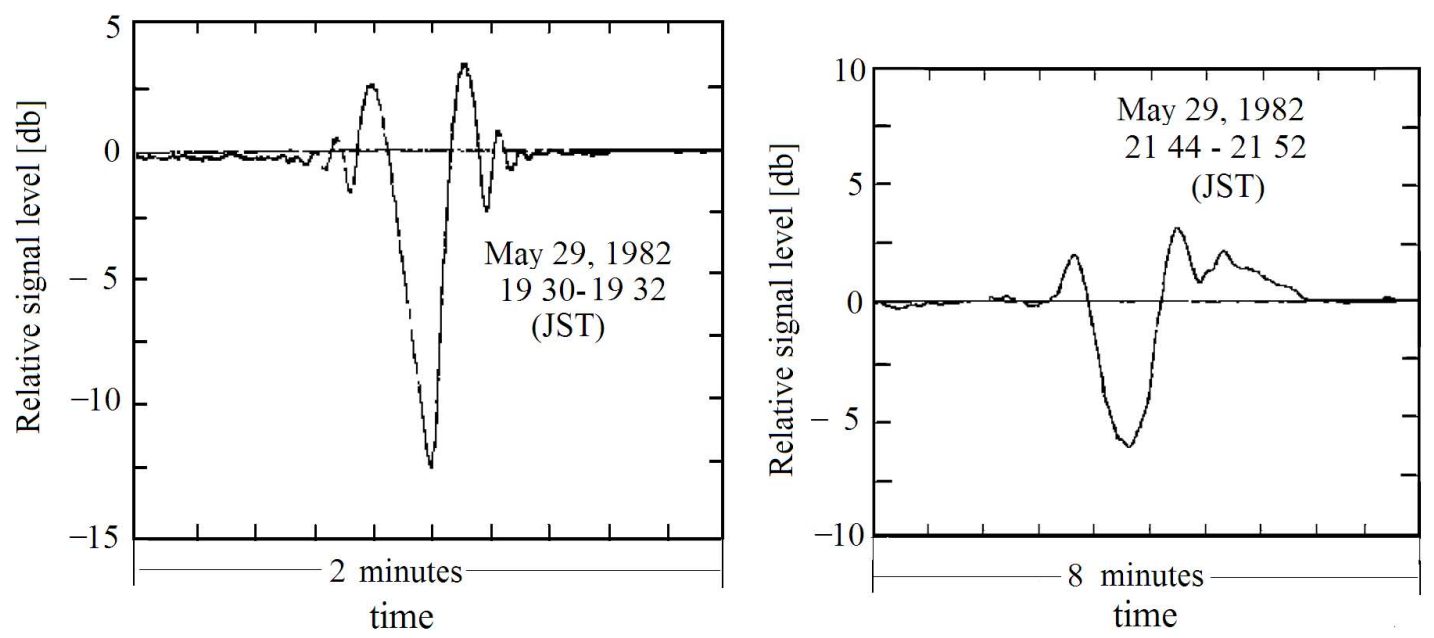

Fig. 6. Two examples of S-type variations of the relative signal level in the trans-ionospheric geostationary satellite-Earth communication link at frequency of $1.5415 \mathrm{GHz}$ (Karasawa et al., 1985). 


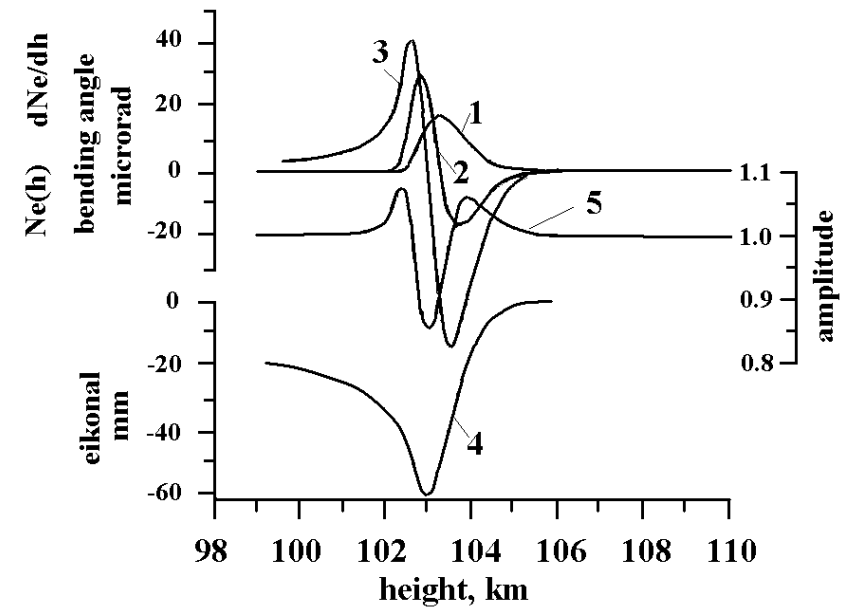

Fig. 7. Simulation of the ionospheric impact on the RO signal as a function of the altitude of the RO ray perigee by the analytical model. Curve 1 and 2 are relevant to the altitude distribution of electron density $N_{\mathrm{e}}(h)\left[10^{3} \mathrm{el} \mathrm{cm}^{-3}\right]$ and its vertical gradient $\frac{d N_{\mathrm{e}}(h)}{d h}$ $\left[10^{3} \mathrm{el} \mathrm{cm}^{-3} \mathrm{~km}^{-1}\right]$, respectively. Curves $3-5$ describe the altitude dependence of the bending angle [microradians], eikonal excess [mm], and amplitude of the RO signal [normalized], respectively.

Figure 9 shows three centres of local spherical symmetry associated with a single $\mathrm{RO}$ ray path in the ionosphere and atmosphere. These centres are located at points $\mathrm{O}_{1}, \mathrm{O}_{2}, \mathrm{O}_{3}$ corresponding to three parts of the ray trajectory $G_{1} B_{1} B_{2}$ $\mathrm{L}$ in three spherical sectors with boundaries at the points $\mathrm{B}_{1}$ and $B_{2}$, respectively: $G_{1} B_{1}$ (the ionosphere between transmitter $\mathrm{G}_{1}$ and atmosphere), $\mathrm{B}_{1} \mathrm{~B}_{2}$ (atmospheric part), and $\mathrm{B}_{2} \mathrm{~L}$ (the ionosphere between the receiver and atmosphere). The points $\mathrm{G}_{1}, \mathrm{O}_{1}, \mathrm{O}_{2}, \mathrm{O}_{3}$, $\mathrm{L}$ are assumed to lie in the $\mathrm{G}_{1} \mathrm{~B}_{1}$ $\mathrm{B}_{2} \mathrm{~L}$ plane.

This geometry corresponds to the experimental RO data indicating a significant bending effect in the plane of propagation. The central angles $\theta_{1}, \theta_{2}$, and $\theta_{3}$ between the directions to the transmitter $\mathrm{G}_{1}$ and the receiver $\mathrm{L}$ have vertices located at the points $\mathrm{O}_{1}, \mathrm{O}_{2}, \mathrm{O}_{3}$ (Fig. 9). The distances $\mathrm{G}_{1} \mathrm{O}_{1}$, $\mathrm{G}_{1} \mathrm{O}_{2}, \mathrm{G}_{1} \mathrm{O}_{3}$ and $\mathrm{LO}_{1}, \mathrm{LO}_{2}, \mathrm{LO}_{3}$ are equal to $\mathrm{D}_{1}, \mathrm{D}_{2}, \mathrm{D}_{3}$, and $R_{1}(L), R_{2}(L), R_{3}(L)$, respectively. The points $G_{2}, G_{3}$ (actually $\mathrm{G}_{2}, \mathrm{G}_{3}$ are the images of the transmitter $\mathrm{G}_{1}$ as seen from the points $B_{1}$ and $B_{2}$ ) are intersections of the tangents to the RO ray trajectory $G_{1} B_{1} B_{2} L$ at the points $B_{1}$ and $B_{2}$ with the straight lines $\mathrm{G}_{1} \mathrm{O}_{1}$ and $\mathrm{G}_{2} \mathrm{O}_{2}$, respectively. The angles $\mu_{1}, \mu_{2}$ and $\mu_{3}, \mu_{4}$ have common vertices at the points $\mathrm{G}_{2}$ and $\mathrm{G}_{3}$. The variables $\mu_{1}, \mu_{2}$ are the angles between the tangent to the ray trajectory $G_{1} B_{1} B_{2} L$ at the point $B_{2}$ and the directions of $\mathrm{G}_{2} \mathrm{O}_{1}$ and $\mathrm{G}_{2} \mathrm{O}_{2}$, respectively (Fig. 9). The variables $\mu_{3}, \mu_{4}$ are the angles between the tangent to the ray trajectory $\mathrm{G}_{1} \mathrm{~B}_{1} \mathrm{~B}_{2} L$ at point $\mathrm{B}_{1}$ and the directions $\mathrm{G}_{3} \mathrm{O}_{2}$, $\mathrm{G}_{3} \mathrm{O}_{3}$, accordingly (Fig. 9). Dependence of the excess phase path (eikonal) and refractive attenuation on the impact parameter $p$ can be considered separately for three parts of the
RO ray trajectory $G_{1} B_{1} B_{2} L$ (Fig. 9). In the general case of $N$ spherically symmetric sectors the eikonal $\Phi$ corresponding to the ray $\mathrm{G}_{1}$ L (Fig. 9) is a sum (Pavelyev et al., 2010b):

$$
\begin{aligned}
\Phi & =\sqrt{D_{1}^{2}-p_{1}^{2}}+\sqrt{R_{N}^{2}-p_{N}^{2}} \\
& +\sum_{i=1}^{i=N-1} b_{i} \cos \left(\gamma_{i}-\xi_{i}-\alpha_{i}\right) \\
& +\sum_{m=1}^{N}\left[p_{m} \xi_{m}\left(p_{m}\right)+\kappa_{m}\left(p_{m}\right)\right]
\end{aligned}
$$

where $p_{i}, \xi_{i}\left(p_{i}\right), \kappa_{i}\left(p_{i}\right)$ are the impact parameter, the bending angle, and the main refractivity part of the phase path corresponding to the $i$-th spherical sector. Due to spherical symmetry, the impact parameters $p_{1}, p_{2}, \ldots, p_{N}$ satisfy the following relationships, which are valid inside the $i$-th spherical sector:

$p_{i}=n\left(R_{i}\right) R_{i} \sin \gamma_{\mathrm{e}}, i=1, \ldots, N$

where $n\left(R_{i}\right)$ is the refractive index, $\gamma_{\mathrm{e}}$ is the angle between the tangent to the ray trajectory $\mathrm{G}_{1} \mathrm{~L}$ at the current point $E$ and direction to the center of a spherical sector. The tangents to the ray trajectory $\mathrm{G}_{1} \mathrm{~L}$ and directions to the centers $\mathrm{O}_{1}, \mathrm{O}_{2}, \ldots, \mathrm{O}_{N}$ make the angles $\gamma_{1}, \gamma_{2}, \ldots, \gamma_{N}$ at the point $\mathrm{G}_{1}$ and $\beta_{1}, \beta_{2}, \ldots, \beta_{N}$ at the point $\mathrm{L}$, respectively. The relationships between the impacts $p_{1}, p_{2}, \ldots, p_{N}$ central angles $\theta_{1}, \theta_{2}, \ldots, \theta_{N} \mathrm{~s}$ and bending angles $\xi_{1}, \xi_{2}, \ldots, \xi_{N}$ can be obtained using the geometry of the ray path $\mathrm{G}_{1} \mathrm{~L}$ :

$p_{i+1}=p_{i}+b_{i} \sin \left(\gamma_{i}-\alpha_{i}-\sum_{k=1}^{i} \xi_{k}\right) ; i=1, \ldots, N-1$

$\theta_{i}=\pi+\xi\left(p_{1}\right)-\gamma_{i}-\beta_{i}, \xi\left(p_{1}\right)=\sum_{i=1}^{i=N} \xi_{i}\left(p_{i}\right)$

The method introduced previously (Pavelyev and Kucherjavenkov, 1978) for the case of a spherically symmetric medium has been used by Pavelyev et al. (2010b) in the geometrical optics approximation in the general case of a medium consisting of $N$ spherically symmetric sectors to obtain an exact expression for the refractive attenuation of electromagnetic waves $X_{\mathrm{L}}$ :

$X_{\mathrm{L}}=R_{0}^{2} \sin \gamma_{1} /\left[d R_{i}(\mathrm{~L}) \cos \beta_{i}\left|\partial \theta_{i} / \partial \gamma_{1}\right| d \mathrm{~S}(1) \ldots \mathrm{S}(\mathrm{N}-1)\right], i=1, \ldots, N ;$

$\mathrm{S}(\mathrm{i})=\sin \mu_{2 i-1} / \sin \mu_{2 i}, \mathrm{~S}(0)=1, d=R_{N}(\mathrm{~L}) \sin \Theta$

Relationships (4-8) represent the core of the analytical model. The phase path and the refractive attenuation depend, respectively, on the sum of the phase changes and the bending angles in the spherical sectors and practically do not depend on the location of their boundaries. Any intense locally spherically symmetric layer along the ray in the ionosphere can produce unexpected strong variations in the amplitude and phase of the RO signals at the $40-90 \mathrm{~km}$ altitudes of the 

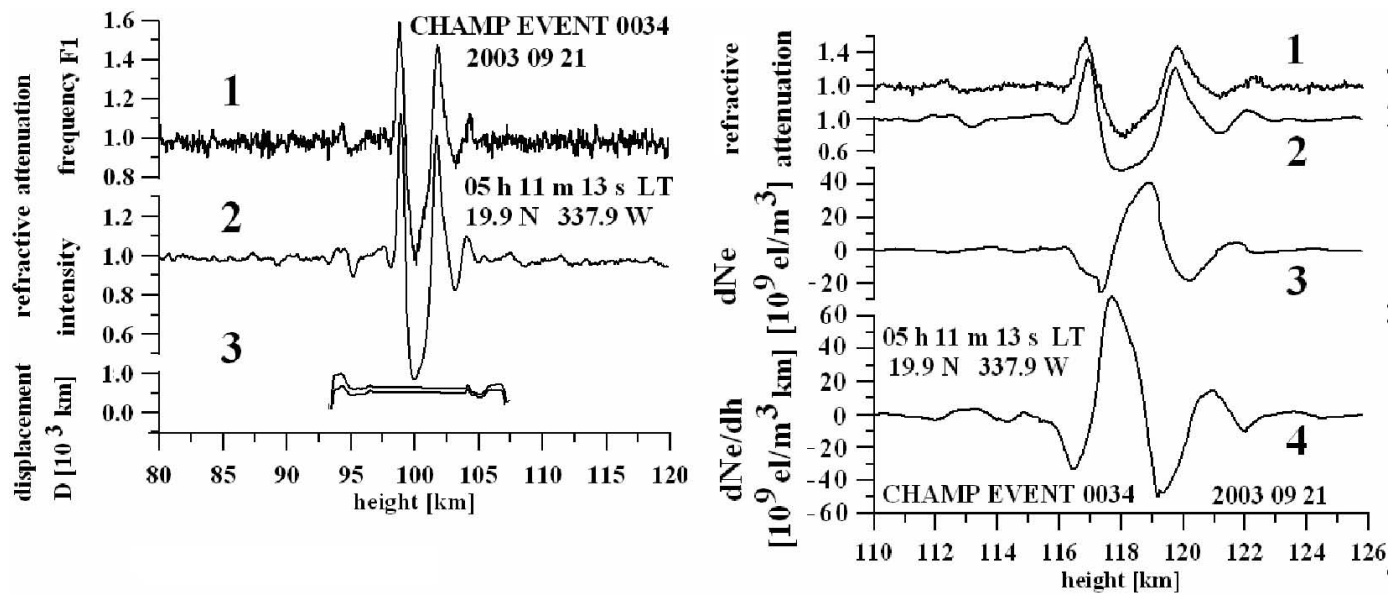

Fig. 8. Comparison of the refractive attenuation recalculated from the excess phase path data $X_{\mathrm{p}}$, and refraction attenuation $X_{\mathrm{a}}$ found from the amplitude data at the first GPS frequency $f_{1}$ (curves 1 and 2), results of estimation of distance $d$ from the RO ray perigee (curve 3 ) (left panel) and retrieved variations of the electron density and its gradients (curves 3 and 4) (right panel).

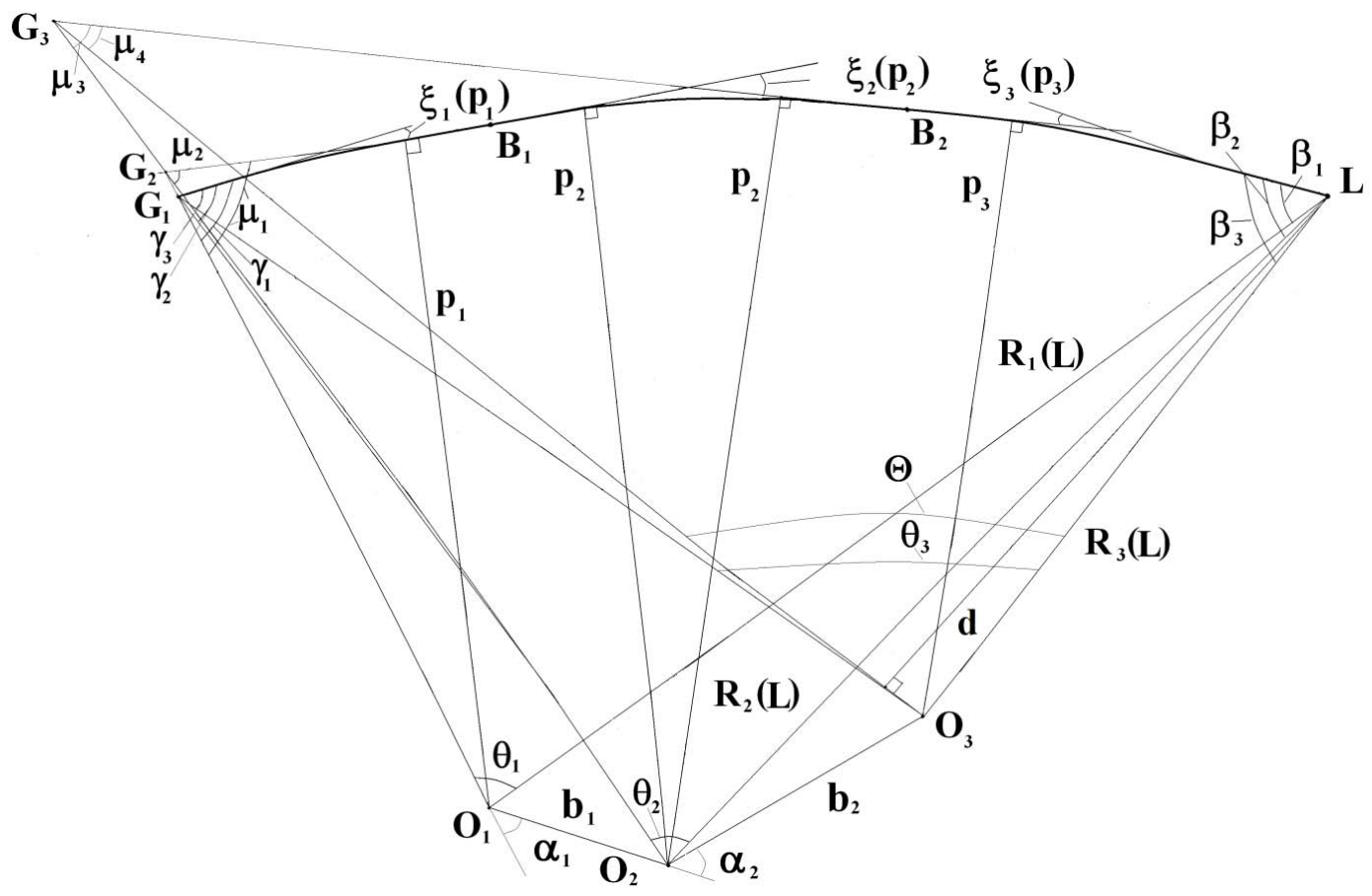

Fig. 9. Geometry of electromagnetic wave propagation through three spherical symmetric sectors. The points $\mathrm{G}_{1}, \mathrm{~B}_{1}, \mathrm{~B}_{2}, \mathrm{~L}$ are supposed to be located in the same plane with the centers of spherical symmetry $\mathrm{O}_{1}, \mathrm{O}_{2}, \mathrm{O}_{3}$. The points $\mathrm{G}_{2}, \mathrm{G}_{3}$ are intersections of the tangents to the ray trajectory $G_{1} B_{1} B_{2} L$ at the points $B_{1}, B_{2}$ with the straight lines $G_{1} O_{1}$ and $G_{2} O_{2}$ respectively. The angle $\Theta$ has vertex at point $\mathrm{O}_{3}$. The value $\Theta$ determines the angle between the directions $\mathrm{G}_{3} \mathrm{O}_{3}$, and $\mathrm{LO}_{3}$.

RO ray perigee. Therefore, the displacement of the tangent point is a main cause of systematic error in the RO estimation of the altitude of inclined ionospheric layers. This conclusion may also be valid in the case of electromagnetic waves propagation in the satellite-to-Earth communication links.
The analytical model can be applied to ray tracing of the RO signals. If the impact parameter $p_{1}$ is known, then one can successively determine the impact parameters $p_{i}, i=$ $2, \ldots, N$ and the bending angle $\xi\left(p_{1}\right)$ from Eqs. (6), (7), and recalculate the phase path $\Phi\left(p_{1}\right)$ and the refractive attenuation $X_{\mathrm{L}}$ from Eqs. (4) and (8). Note the following important feature of the model. If the bending effect is absent in the 
$k$-th sector, then the dimension $N$ of model can be reduced to $N-1$. In this case the following equality holds:

$\mu_{2 k-1}=\mu_{2 k} ; \mathrm{S}(k)=1$

and the refractive attenuation Eq. (8) does not depend on the contribution of the $k$-th spherical sector.

The phase path of electromagnetic waves transmitted through the atmosphere can be represented by formula Eq. (4) as a linear function of the bending angles in the corresponding sectors if the bending effect is small. Therefore, the linear methods of ionospheric correction introduced earlier (Melbourne et al., 1994; Vorob'ev and Krasilnikova, 1994) should be effective in the case of propagation through several spherically symmetric sectors in the case of undisturbed ionosphere. However, the amplitude of the RO signal is a nonlinear function of the bending angle and, in the case of disturbed ionosphere, is subject to strong perturbations.

Let us consider the refractive attenuation in the particular case when the bending effect exists in the first sector due to the ionospheric influence and is absent in the remaining sectors. This is typical situation when the amplitude and phase variations are observed at the altitudes $40-90 \mathrm{~km}$ where the neutral atmosphere influence is minimal. In this case the points $\mathrm{G}_{2}, \ldots, G_{N}$ coincide. As a consequence, the following relationship is fulfilled:

$\mu_{3}=\mu_{2}, \mu_{5}=\mu_{4}, \ldots, \mu_{2 N-3}=\mu_{2 N-4}$

After substitution of Eq. (10) into Formula (8), one can obtain

$X_{L}=R_{0}^{2} \sin \gamma_{1} /\left[R_{1}^{2}(\mathrm{~L}) \cos \beta_{1}\left|\partial \theta_{1} / \partial \gamma_{1}\right| \sin \theta_{1}(\mathrm{~L})\right]$

Equation (11) has been obtained previously (Pavelyev and Kucherjavenkov, 1978). Equations (5), (7), and (11) imply a simple formula for the refractive attenuation $X_{\mathrm{L}}$ (Pavelyev et al., 2004):

$$
X_{\mathrm{L}}=\frac{p_{1} R_{0}}{p_{\mathrm{s}} \sqrt{R_{1}^{2}-p_{1}^{2}} \sqrt{R_{2}^{2}-p_{1}^{2}\left|\frac{\partial \theta_{1}}{\partial p_{1}}\right|}}=\frac{p_{1}}{p_{\mathrm{s}}\left|1-\frac{\sqrt{R_{1}^{2}-p_{1}^{2}}}{R_{0}} \sqrt{R_{2}^{2}-p_{1}^{2}} \frac{d \xi_{1}}{\mathrm{~d} p_{1}}\right|}
$$

where $p_{\mathrm{S}}$ is the impact parameter corresponding to the straight line GL (Fig. 1).

The analytical model introduced provides a simple explanation for the strong amplitude and phase variations of the RO signals at the $30-90 \mathrm{~km}$ altitudes of the RO ray perigee by relating them to the displacement of the center of spherical symmetry in the ionosphere. This allows one to estimate the inclination of plasma layers as shown by Wickert et al. (2004) and gives evidences and support to their hypothesis on the origin of the regular variations of the RO signal in the 30-90 km interval of the RO ray perigee as connected with the refractive attenuation in inclined ionospheric layers.

In short, the introduced analytical model is characterized as threefold:
1. (i) In the simplest case, when an inclined plasma layer exists only in one sector and the influence of the neutral atmosphere is weak, the analytical model predicts the displacement of the tangent point from the ray perigee $T$ to a plasma layer. The Abel transformation can be applied to the inversion of the RO ionospheric data, including the case when the altitudes $h$ of the RO ray perigee are below $90 \mathrm{~km}$. Due to spherical symmetry, one can estimate the electron density distribution in inclined ionospheric plasma layers.

2. (ii) A difficulty exists to locate plasma layers and determine their altitude.

3. (iii) Analytical model can be used for analytical ray tracing because it gives analytical expressions for the eikonal, bending angle, and refractive attenuation in general case of a medium consisting of locally spherically symmetric sectors.

\section{Identification and location of plasma layers}

Let us assume that one of the spherical sectors, for example, the first one, is located in the ionosphere with centre at point $\mathrm{O}_{1}$ (Fig. 9) and introduces the most impact on the radio wave propagating along the ray GTL. In this case $N=1$, expressions for the excess phase and the central angle $\theta$ (Eqs. 4, 5, 7) can be rewritten as:

$$
\begin{aligned}
\Phi(t) & =\sqrt{D_{1}^{2}-p^{2}}+\sqrt{R_{1}^{2}(\mathrm{~L})-p^{2}} \\
& +p \xi(p)+\kappa_{1}(p)-R_{0}, \\
p & =p_{1}, \xi(p)=\xi_{1}(p) ; R_{0}=\sqrt{D_{1}^{2}-p_{\mathrm{s}}^{2}} \\
& +\sqrt{R_{1}^{2}(\mathrm{~L})-p_{\mathrm{s}}^{2}} ; \xi_{1}(p)=-\frac{d \kappa_{1}(p)}{\mathrm{d} p} ;
\end{aligned}
$$

$$
\begin{aligned}
\theta & =\pi+\xi_{1}(p)-\sin ^{-1} \frac{p}{D_{1}}-\sin ^{-1} \frac{p}{R_{1}(\mathrm{~L})} \\
& =\pi-\sin ^{-1} \frac{p_{\mathrm{s}}}{D_{1}}-\sin ^{-1} \frac{p_{\mathrm{s}}}{R_{1}(\mathrm{~L})}, n\left(D_{1}\right) \\
& =n\left(R_{1}(\mathrm{~L})\right)=1
\end{aligned}
$$

where $R_{0}, R_{1}(\mathrm{~L})$ are the distances GL and $\mathrm{LO}_{1}$, and $p_{\mathrm{s}}$ is the impact parameter equal to the distance between the centre $\mathrm{O}_{1}$ and the line of sight GL (Fig. 9). For convenience in the notations $\xi_{1}\left(p_{1}\right)$ and $p_{1}$ the index is removed (Eqs. 13 and 14). From Eqs. (13) and (14), one can obtain by differentiating the excess phase path $\Phi(t)$ and the central angle $\theta$ with respect to time $t$ an exact relationship that connects the 
Doppler frequency $F_{\mathrm{d}}(t)$ of the RO signal with the impact parameters $p, p_{\mathrm{s}}$ (Liou et al. 2007):

$$
\begin{aligned}
& \lambda F_{\mathrm{d}}(t)=\frac{d \Phi(t)}{\mathrm{d} t}=-\left(p-p_{\mathrm{s}}\right) \\
& {\left[\left(d_{1 \mathrm{~s}}^{-1}+d_{2 \mathrm{~s}}^{-1}\right) \frac{\mathrm{d} p_{\mathrm{s}}}{\mathrm{d} t}+\left(p^{2}-p_{\mathrm{s}}^{2}\right) \sum_{i=1}^{i=2} Y_{i} \frac{d Y_{i}}{\mathrm{~d} t} M_{i}\right]} \\
& M_{i}=\left[d_{i \mathrm{~s}}\left(d_{i \mathrm{~s}}+d_{i}\right)\left(p d_{i \mathrm{~s}}+p_{\mathrm{s}} d_{i}\right)\right]^{-1} \\
& Y_{1}=D_{1} ; Y_{2}=R_{1}(\mathrm{~L}) ; d_{i}=\sqrt{Y_{i}^{2}-p^{2}} \\
& d_{i \mathrm{~s}}=\sqrt{Y_{i}^{2}-p_{\mathrm{s}}^{2}} ; i=1,2
\end{aligned}
$$

where $\lambda$ is the wavelength. Equation (15) is valid in the geometrical optics approximation when the multipath propagation is absent. Equation (15) can be simplified under the condition

$$
\left|\left(d_{1 \mathrm{~s}}^{-1}+d_{2 \mathrm{~s}}^{-1}\right) \frac{\mathrm{d} p_{\mathrm{s}}}{\mathrm{d} t}\right| \gg\left|\left(p^{2}-p_{\mathrm{s}}^{2}\right) \sum_{i=1}^{i=2} Y_{i} \frac{d Y_{i}}{\mathrm{~d} t} M_{i}\right|
$$

This inequality is valid in most practical RO situations. Under condition (16), one can obtain from Eq. (15) the following approximate formula for estimating the difference $p-p_{\mathrm{s}}$ on the Doppler frequency $F_{\mathrm{d}}(t)$ :

$\lambda F_{\mathrm{d}}(t)=\frac{d \Phi(t)}{\mathrm{d} t}=-\left(p-p_{\mathrm{s}}\right)\left(d_{1 \mathrm{~s}}^{-1}+d_{2 \mathrm{~s}}^{-1}\right) \frac{\mathrm{d} p_{\mathrm{s}}}{\mathrm{d} t}$

There exists a relationship between the refractive attenuation $X_{\mathrm{L}}$ and the time derivative of the impact parameter $p$ (Kalashnikov et al. 1986; Liou et al., 2006). This relationship can be obtained from Eq. (14) by differentiating the central angle $\theta$ on time with regard to Eq. (12):

$$
\begin{aligned}
& X_{\mathrm{L}}\left[\frac{\partial \theta}{\partial p_{\mathrm{s}}} \frac{\mathrm{d} p_{\mathrm{s}}}{\mathrm{d} t}+R_{1}^{-1}(\mathrm{~L}) \frac{\mathrm{dR}_{1}(\mathrm{~L})}{\mathrm{d} t}\left(\frac{p_{\mathrm{s}}}{d_{2 \mathrm{~s}}}-\frac{p}{d_{2}}\right)\right. \\
& \left.+D_{1}^{-1} \frac{d D_{1}}{\mathrm{~d} t}\left(\frac{p_{\mathrm{s}}}{d_{1 \mathrm{~s}}}-\frac{p}{d_{1}}\right)\right]=\frac{p R_{0}}{d_{1} d_{2} p_{\mathrm{s}}} \frac{\mathrm{d} p}{\mathrm{~d} t} \operatorname{sign}\left(\frac{\partial \theta}{\partial p}\right)
\end{aligned}
$$

Under conditions:

$$
\begin{aligned}
& \left|p-p_{\mathrm{s}}\right| \ll p, p_{\mathrm{s}} ; \mid R_{1}^{-1}(\mathrm{~L}) \frac{d R_{1}(\mathrm{~L})}{\mathrm{d} t}\left(\frac{p_{\mathrm{s}}}{d_{2 \mathrm{~s}}}-\frac{p}{d_{2}}\right) \\
& +D_{1}^{-1} \frac{d D_{1}}{\mathrm{~d} t}\left(\frac{p_{\mathrm{s}}}{d_{1 \mathrm{~s}}}-\frac{p}{d_{1}}\right)|\ll| \frac{\partial \theta}{\partial p_{\mathrm{s}}} \frac{\mathrm{d} p_{\mathrm{s}}}{\mathrm{d} t} \mid
\end{aligned}
$$

which are valid practically in most radio occultation events, one can obtain from Eq. (18)

$\frac{\mathrm{d} p}{\mathrm{~d} t}=X_{\mathrm{L}} \frac{\mathrm{d} p_{\mathrm{s}}}{\mathrm{d} t}$

In the case of the global spherical symmetry of the ionosphere, the points $\mathrm{O}_{1}$ and $\mathrm{O}_{2}$ coincide. In this case parameters $p_{\mathrm{s}}, \frac{d p_{\mathrm{s}}}{\mathrm{d} t}, d_{i \mathrm{~s}}$ can be derived from the orbital data. The time differentiation of both sides of Eq. (17) with regard to Eq. (20) yields:

$$
\begin{aligned}
& \lambda \frac{d F_{\mathrm{d}}(t)}{\mathrm{d} t}=\frac{d^{2} \Phi(t)}{\mathrm{d} t^{2}}=-\left(\frac{\mathrm{d} p}{\mathrm{~d} t}-\frac{\mathrm{d} p_{\mathrm{s}}}{\mathrm{d} t}\right) \\
& \left(d_{1 \mathrm{~s}}^{-1}+d_{2 \mathrm{~s}}^{-1}\right) \frac{\mathrm{d} p_{\mathrm{s}}}{\mathrm{d} t}=\left(1-X_{\mathrm{L}}\right)\left(d_{1 \mathrm{~s}}^{-1}+d_{2 \mathrm{~s}}^{-1}\right) \frac{\mathrm{d} p_{\mathrm{s}}}{\mathrm{d} t}
\end{aligned}
$$

Relationship (21) is valid under the conditions

$$
\begin{aligned}
& \left|p-p_{\mathrm{s}}\right| \ll p, p_{\mathrm{s}} ;\left|\left(p-p_{\mathrm{s}}\right) \frac{d\left[\left(d_{1 \mathrm{~s}}^{-1}+d_{2 \mathrm{~s}}^{-1}\right) \frac{\mathrm{d} p_{\mathrm{s}}}{\mathrm{d} t}\right]}{\mathrm{d} t}\right| \\
& \ll\left|\left(\frac{\mathrm{d} p}{\mathrm{~d} t}-\frac{\mathrm{d} p_{\mathrm{s}}}{\mathrm{d} t}\right)\left(d_{1 \mathrm{~s}}^{-1}+d_{2 \mathrm{~s}}^{-1}\right) \frac{\mathrm{d} p_{\mathrm{s}}}{\mathrm{d} t}\right|
\end{aligned}
$$

One can obtain from Eq. (21) the following relationships, which have been derived and analyzed earlier by Liou and Pavelyev (2006); Liou et al. (2007):

$$
\begin{aligned}
& 1-X_{\mathrm{L}}(t)=m a=\frac{m d\left(\lambda F_{\mathrm{d}}\right)}{\mathrm{d} t}=m \frac{d^{2} \Phi(p)}{\mathrm{d} t^{2}} \\
& m=q\left(\frac{\mathrm{d} p_{\mathrm{s}}}{\mathrm{d} t}\right)^{-2} ; q=d_{2 \mathrm{~s}}\left(1-d_{2 \mathrm{~s}} R_{0}^{-1}\right) ; d_{2 \mathrm{~s}}=\sqrt{R_{1}^{2}(\mathrm{~L})-p_{\mathrm{s}}^{2}}
\end{aligned}
$$

where $R_{0}$ is the distance along the straight line GL. Note that the distance $d_{2 \mathrm{~s}}$ is nearly equal to distance TL (Fig. 1) within an accuracy according to the horizontal resolution of the RO method (about 100-300 km). Parameters $m$ and $\mathrm{d} p_{\mathrm{s}} / \mathrm{d} t$ can be evaluated using the orbital data.

In the case of global spherical symmetry, one can use an analytical model of refractivity, bending angle, and refractive attenuation $X_{m}$ to simplify the inversion technique and employ a perturbation method to find the deviations from the model. In the same way, one can obtain the following relationship:

$1-X_{m}(t)=m a_{m}=m d\left(\lambda F_{\mathrm{d} m}\right) / \mathrm{d} t=m d^{2} \Phi_{m}(p) / \mathrm{d} t^{2}$

where $a_{m}, \Phi_{m}, F_{d m}$ are the eikonal acceleration, excess phase, and Doppler frequency corresponding to the model of refractivity. Subtracting both sides of the first Eqs. (23) and (24) gives:

$$
\begin{aligned}
X_{L}(t)-X_{m}(t) & =m\left(a_{m}-a\right)=\lambda m \frac{d\left(F_{\mathrm{d} m}-F_{\mathrm{d}}\right)}{\mathrm{d} t} \\
& =m \frac{d^{2}\left[\Phi_{m}(p)-\Phi(p)\right]}{\mathrm{d} t^{2}}
\end{aligned}
$$

Equation (25) can be applied to find the features of the vertical profiles of refractivity by a perturbation method.

Two important conclusions follow from Eq. (23):

(i) The refractive attenuation $X_{\mathrm{p}}(t)$ can be recalculated from the right-hand side of the first Eq. (23) by using known values of the eikonal acceleration $a$ and parameter $m$ :

$1-X_{\mathrm{p}}(t)=m a$

One can estimate the integral absorption $\Gamma$ in the atmosphere (or ionosphere) using the attenuation $X_{\mathrm{a}}(t)$ found from the amplitude variations of the RO signals via equations (Pavelyev et al., 2009):

$\Gamma=1-\frac{X_{\mathrm{a}}(t)}{X_{\mathrm{p}}(t)} ; X_{\mathrm{a}}(t)=\frac{I}{I_{0}}$

where $I, I_{0}$ are the intensities of radio waves in the atmosphere (ionosphere) and in free space, respectively. When 

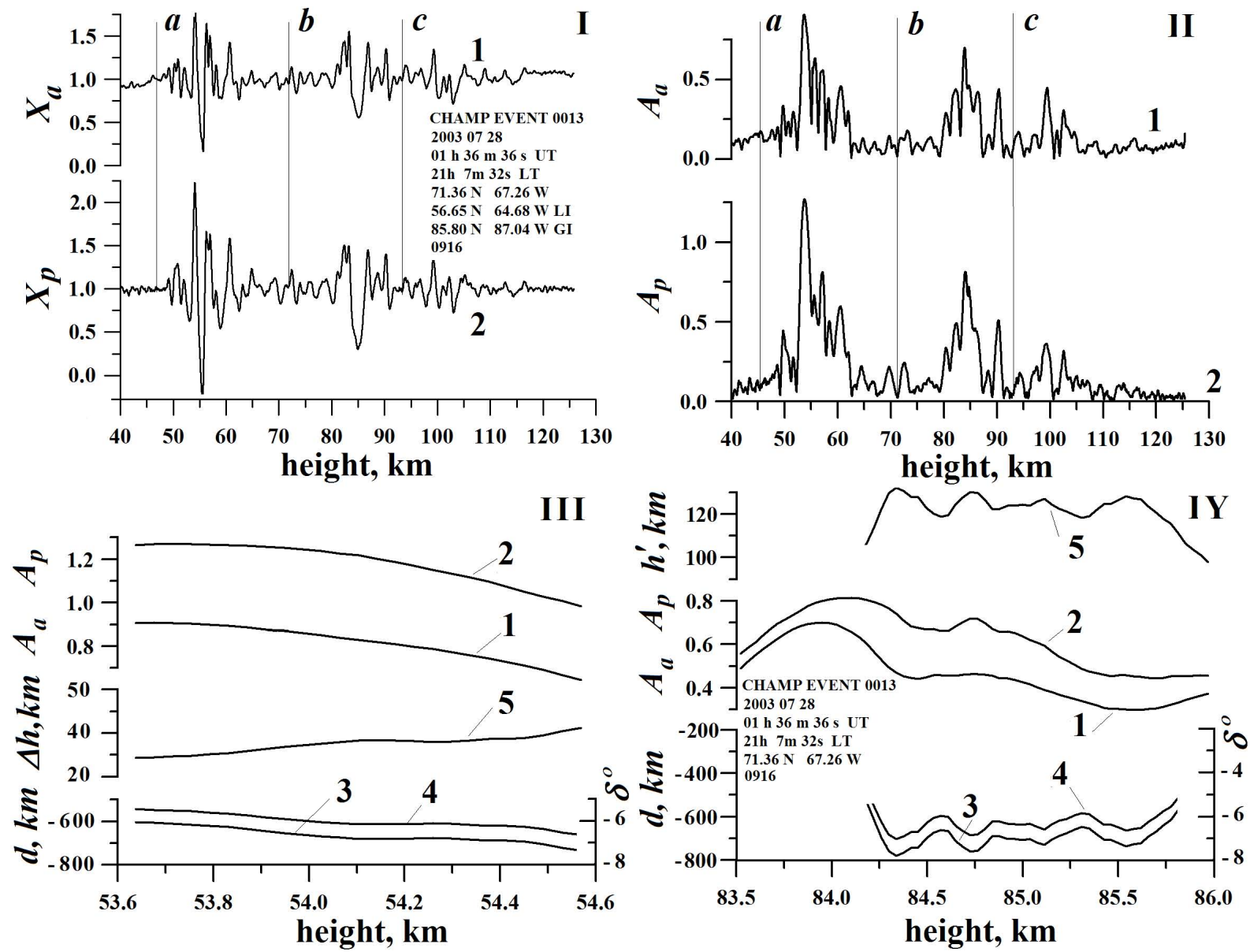

Fig. 10. I. Comparison of the refractive attenuations $X_{\mathrm{a}}, X_{\mathrm{p}}$ found from the RO intensity and eikonal data at GPS frequency $f_{1}$ (curves 1 and 2 , respectively). II. The amplitudes of analytical signals corresponding to the variations of the refractive attenuations $X_{\mathrm{a}}, X_{\mathrm{p}}$ (curves 1 and 2). III. Location of the first layer using amplitudes $A_{\mathrm{a}}, A_{\mathrm{p}}$. IY. Location of the second layer using amplitudes $A_{\mathrm{a}}, A_{\mathrm{p}}$.

$\Gamma=0$ the following identity can be obtained using Eqs. (23) and (27):

$X_{\mathrm{p}}(t) \equiv X_{\mathrm{a}}(t)$

Identity Eq. (28) represents a criterion which is necessary and sufficient for a layer to be located at the $\mathrm{RO}$ ray perigee $T$ (Fig. 1).

(ii) When the variations of the refractive attenuations $1-$ $X_{\mathrm{p}}(t)$ and $1-X_{\mathrm{a}}(t)$ can be described by narrowband oscillating functions, they can be presented in the analytical forms:

$1-X_{\mathrm{p}}(t)=m a=A_{\mathrm{p}}(t) \operatorname{Re}\left[\exp j \chi_{\mathrm{p}}(t)\right] ;$

$1-X_{\mathrm{a}}(t)=A_{\mathrm{a}}(t) \operatorname{Re}\left[\exp j \chi_{\mathrm{a}}(t)\right]$

where $A_{\mathrm{p}}(t), A_{\mathrm{a}}(t) ; \chi_{\mathrm{p}}(t), \chi_{\mathrm{a}}(t)$ are, respectively, the amplitude and phase of the analytical signals, corresponding to $1-X_{\mathrm{p}}(t)$ and $1-X_{\mathrm{a}}(t)$. In this case, one can formulate an analytical criterion for the location of a layer in the RO ray perigee:

$A_{\mathrm{p}}(t)=A_{a}(t), \chi_{\mathrm{a}}(t)=\chi_{\mathrm{p}}(t)$
Deviations from this criterion can be caused by the displacement of the centre of spherical symmetry, multipath propagation, diffraction, scattering, influence of turbulence, and other irregularities in the atmosphere and ionosphere.

The ionospheric layers can have different location of the centre of spherical symmetry (Fig. 1). In this case the inequalities Eqs. (16), (19) and Formulas (17), (20) remain valid, but the impacts $p_{\mathrm{s}}, p$ need to be replaced with values $p_{\mathrm{s}}^{\prime}, p^{\prime}$ corresponding to new centre $\mathrm{O}^{\prime}$ (Fig. 1).

The criteria (28) and (30) are also valid in the new coordinate system with the centre at the point $\mathrm{O}^{\prime}(\mathrm{Fig}$. 1) with the refractive attenuations $X_{\mathrm{p}, \mathrm{a}}$, amplitudes $A_{\mathrm{p}, \mathrm{a}}$, and phases $\chi_{\mathrm{p}, \mathrm{a}}$ replaced by $X_{\mathrm{p}, \mathrm{a}}^{\prime}, A_{\mathrm{p}, \mathrm{a}}^{\prime}$, and $\chi_{\mathrm{p}, \mathrm{a}}^{\prime}$, respectively. The measured refractive attenuation $X_{\mathrm{a}}(t)$ found from the amplitude data does not depend on location of the spherical symmetry centre: $X_{\mathrm{a}}^{\prime}=X_{\mathrm{a}}, A_{\mathrm{a}}^{\prime}=A_{\mathrm{a}} ; \chi_{\mathrm{a}}^{\prime}=\chi_{\mathrm{a}}$, and criterion (28) can be represented in a new form:

$X_{\mathrm{p}}^{\prime}(t) \equiv X_{\mathrm{a}}(t)$ 
Equation (23) can be rewritten as:

$1-X_{\mathrm{a}}(t)=1-X_{\mathrm{p}}^{\prime}(t)=m^{\prime} a, a=\frac{d^{2} \Phi(t)}{\mathrm{d} t^{2}} ;$

$m^{\prime}=d_{2 \mathrm{~s}}^{\prime}\left(1-d_{2 \mathrm{~s}}^{\prime} R_{0}^{-1}\right)\left(\frac{\mathrm{d} p_{\mathrm{s}}^{\prime}}{\mathrm{d} t}\right)^{-2} ; d_{2 \mathrm{~s}}^{\prime}=\sqrt{R_{1}^{2}(\mathrm{~L})-p_{\mathrm{s}}^{\prime 2}}$

where $m^{\prime}$ is a new value of the parameter $m$ corresponding to the center of spherical symmetry $\mathrm{O}^{\prime}$. As compared with Formula (23), the Eq. (32) is different with new values of the refractive attenuation $X_{\mathrm{p}}^{\prime}(t)$ and parameter $m^{\prime}$. It follows from criterion (31) that the functions $A_{\mathrm{p}}^{\prime}(t)$ and $A_{\mathrm{p}}(t)$ are connected with $A_{\mathrm{a}}(t)$ by the relationships:

$A_{\mathrm{p}}^{\prime}(t)=\frac{m^{\prime}}{m} A_{\mathrm{p}}(t)=A_{\mathrm{a}}(t) ; A_{\mathrm{p}}(t)=\frac{m}{m^{\prime}} A_{\mathrm{a}}(t)$

Under conditions

$\frac{d_{2 \mathrm{~s}}}{R_{0}}, \frac{d_{2 \mathrm{~s}}^{\prime}}{R_{0}} \ll 1 ; \frac{\mathrm{d} p_{\mathrm{s}}}{\mathrm{d} t} \approx \frac{\mathrm{d} p_{\mathrm{s}}^{\prime}}{\mathrm{d} t}$

the last Eq. (33) can be presented as

$A_{\mathrm{a}}(t)=\frac{d_{2 \mathrm{~s}}^{\prime}}{d_{2 \mathrm{~s}}} A_{\mathrm{p}}(t)$

According to Eq. (35), the distance $d$ between the RO ray perigee $T$ and new tangent point $T^{\prime} d$ (Fig. 1) depends on the amplitudes $A_{\mathrm{a}}(t)$ and $A_{\mathrm{p}}(t)$ :

$d=d_{2 \mathrm{~s}}{ }^{\prime}-d_{2 \mathrm{~s}}=d_{2 \mathrm{~s}} \frac{A_{\mathrm{a}}-A_{\mathrm{p}}}{A_{\mathrm{p}}} ;$

$d_{2 \mathrm{~s}}=\sqrt{R_{2}^{2}-p_{\mathrm{s}}^{2}} ; R_{2}=R_{1}(\mathrm{~L})$

where $d_{2 \mathrm{~s}}, d_{2 \mathrm{~s}}{ }^{\prime}$ are the distances $D \mathrm{~L}, D^{\prime} \mathrm{L}$, respectively (Fig. 1). As follows from Eq. (36), location of a tangent point on the ray trajectory can be found using the amplitudes $A_{\mathrm{a}, \mathrm{p}}$. The displacement $d$ is positive or negative depending on the sign of the difference $A_{\mathrm{a}}-A_{\mathrm{p}}$; according to this sign the tangent point $T^{\prime}$ is located on the parts GT or TL, respectively. The phases $\chi_{\mathrm{p}}(t), \chi_{\mathrm{a}}(t)$ should be equal within some accuracy determined by the quality of measurements. Note, that Eq. (36) is valid when the distance of one of the satellites from the ray perigee $T$ is many times greater than the corresponding value for the second one. This condition is fulfilled for the planetary radio eclipse experiments provided by the space craft - Earth communication link and GPS occultations (Pavelyev et al., 2009).

An accurate relationship for the distance $d$ has been given previously (Pavelyev et al., 2009):

$d=2 R_{0} \beta\left[1+2 \beta(1-w / v)+(1-4 \beta w / v)^{1 / 2}\right]^{-1}-d_{2}$,

$\beta=m^{\prime} v^{2} / R_{0}, m^{\prime}=\left[1-X_{\mathrm{a}}(t)\right] / a$

where $w$ and $v$ are the velocity components of the GPS and LEO satellites, respectively, which are perpendicular to the straight line GL in the plane GOL (Fig. 1). The components $w$ and $v$ are positive when they directed to the point $\mathrm{O}$ and are negative in the opposite case. The analysis of the CHAMP GPS RO data (Pavelyev et al., 2008 a, b) showed correctness of the relationships (23).

A CHAMP RO event 0013 (28 July 2003, 21:08 LT, $71.4^{\circ} \mathrm{N}, 67.3^{\circ} \mathrm{W}$ ) with strong quasi-regular amplitude and phase variations is used to demonstrate the possibility to locate the plasma layers. The refractive attenuations $X_{\mathrm{a}}, X_{\mathrm{p}}$ of the CHAMP RO signals found from the intensity and eikonal data are shown in Fig. 10 (panel I, curves 1 and 2) as functions of the RO ray perigee altitude $h$. The acceleration $a$ has been estimated numerically by double differentiation of a second order least square polynomial approximation to the RO eikonal data over a sliding time interval $\Delta t(\Delta t=0.5 \mathrm{~s})$. This time interval corresponds approximately to the vertical size of the Fresnel zone of about $1 \mathrm{~km}$ since the vertical component of the radio ray velocity is $\sim 2.1 \mathrm{~km} \mathrm{~s}^{-1}$. The refractive attenuation $X_{\mathrm{p}}$ is derived using Formula (23) with $m$ found from the orbital data. The refractive attenuation $X_{\mathrm{a}}$ has been determined from the $\mathrm{RO}$ amplitude data with averaging over the time interval $\Delta t=0.5 \mathrm{~s}$. The phases of the variables $X_{\mathrm{a}}, X_{\mathrm{p}}$ are strongly connected and the changes of $X_{\mathrm{a}}, X_{\mathrm{p}}$ are primarily caused by three ionospheric layers, whose contributions are shown by letters a, b and c in Fig. 10 (panel I). Sectors a, b and c correspond to the $50-72 \mathrm{~km}, 72-92 \mathrm{~km}$ and $92-116 \mathrm{~km}$ altitude intervals accordingly. The signals $X_{\mathrm{a}}-1, X_{\mathrm{p}}-1$ are coherent in the intervals $\mathrm{a}, \mathrm{b}$ and $\mathrm{c}$. However, their amplitudes are different (Fig. 10, panel I). The amplitudes $A_{\mathrm{a}}, A_{\mathrm{p}}$ related to $X_{\mathrm{a}}-1, X_{\mathrm{p}}-1$ are calculated and shown in Fig. 10, panel II (curves 1 and 2, respectively) using the numerical Hilbert transform. The amplitude $A_{\mathrm{a}}$ in the intervals $\mathrm{a}$ and $\mathrm{b}$ is smaller than the corresponding value $A_{\mathrm{p}}$ (Fig. 10, panel II). The opposite case is observed in the interval c (Fig. 10, panels I and II). The first two layers in the intervals of a and $\mathrm{b}$ have a negative displacement $d$; i.e., two layers are located on the RO ray trajectory between the points $\mathrm{T}$ and $\mathrm{L}$. The upper layer $\mathrm{c}$ is displaced from the RO ray perigee $T$ in the direction to the navigational satellite $\mathrm{G}$ (Fig. 1). These results support the prediction of the analytical model (Sect. 3) on possible changes in the tangent point location caused by locally spherically symmetric sectors in the ionosphere. The method introduced can be used to locate ionospheric layers since the variations of the refractive attenuations $X_{\mathrm{a}}, X_{\mathrm{p}}$ are coherent. The results of estimating the displacement $d$ in the intervals a and $\mathrm{b}$ are shown in Fig. 10 (panels III and IY). Curves 1, 2, and 3 in Fig. 10 (panels III and IY) correspond to the altitude dependence of the amplitudes $A_{\mathrm{a}}, A_{\mathrm{p}}$, and displacement $d$, respectively. Curves 4 in Fig. 10 (panels III and IY) indicate the slope of the layer $\delta$ in degrees (right vertical scales). Curve 5 demonstrates the correction to the altitude $\Delta h[\mathrm{~km}]$ (Fig. 10, panel III) and the actual height $h^{\prime}[\mathrm{km}]$ of a layer (Fig. 10, panel IY), respectively. The changes of $d$ are concentrated in the ranges of $[-630 \mathrm{~km}$, $-800 \mathrm{~km}]$ and $[-600 \mathrm{~km},-750 \mathrm{~km}]$ (intervals $\mathrm{a}, \mathrm{b}$, respectively). The statistical error in the determination of the ratio 

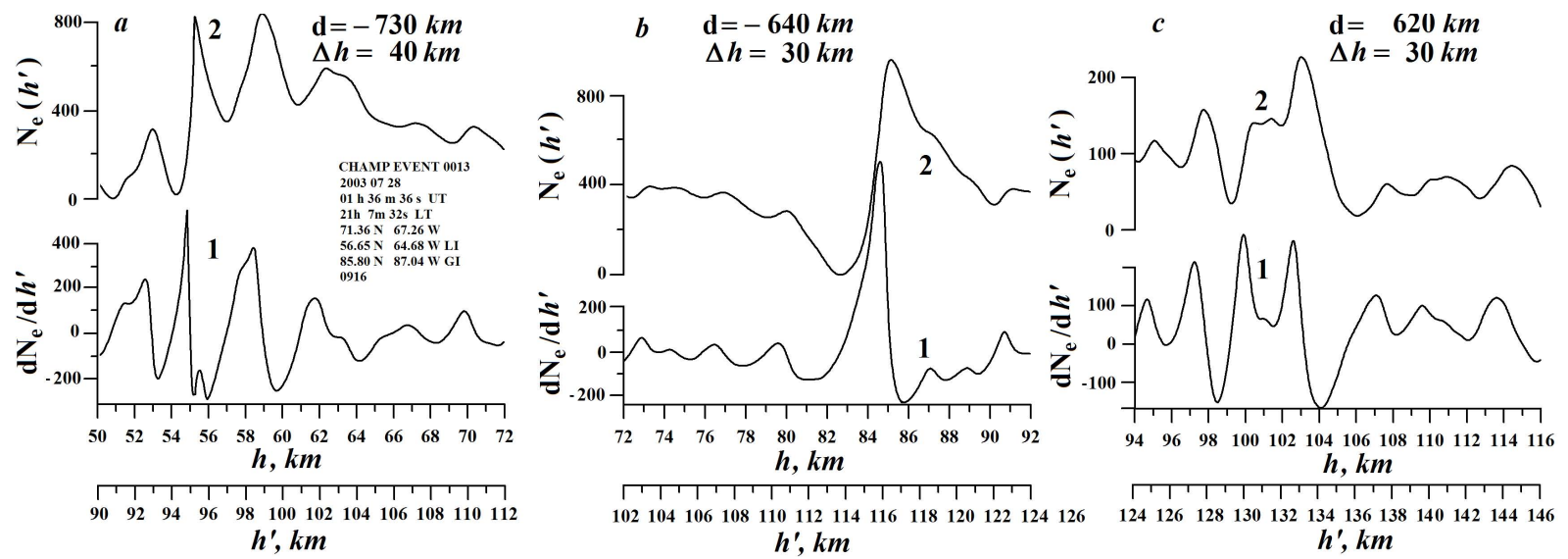

Fig. 11. Restoration of electron density and its vertical gradient.

$\frac{A_{\mathrm{a}}-A_{\mathrm{p}}}{A_{\mathrm{p}}}$ in formula (36) is minimal when $A_{\mathrm{p}}$ is maximal. On average, the estimated values of the displacement in the intervals a, b are equal to $d=-730 \mathrm{~km}$ and $d=-620 \mathrm{~km}$, correspondingly. If the relative error in the measurement of $A_{\mathrm{p}}$ is equal to $5 \%$, then the accuracy in the estimation of $d$ is about $\pm 100 \mathrm{~km}$. The estimated value of the displacement $d$ in the interval $c$ is positive and is equal to $d=620 \mathrm{~km}$. According to formula (3), the inclinations of the plasma layers a, b, and $\mathrm{c}$ to the local horizontal direction are approximately equal to $\delta=-7.3^{\circ} \pm 0.9^{\circ}, \delta=-6.4^{\circ} \pm 0.9^{\circ}$, and $\delta=6.4^{\circ} \pm 0.9^{\circ}$, respectively. The spherical symmetry justifies the application of the Abel transform to solving the inverse problem, i.e., to find the internal distribution of the electron density and its gradient in the layer (Pavelyev et al., 2009).

For the Abel transform the following formula is used (Hocke, 1997):

$N(h)=-\frac{1}{\pi} \int_{p_{0}}^{\infty} \ln \left[\frac{p}{p_{0}}+\sqrt{\left(\frac{p}{p_{0}}\right)^{2}-1}\right] \frac{d \xi(p)}{\mathrm{d} p} \mathrm{~d} p$

where $p_{0}$ is the magnitude of the impact parameter $p$ corresponding to the ray GTL at the initial instant of time $t_{0}$. Using relation (12) between the refractive attenuation $X_{\mathrm{a}}$ and the derivative $\frac{d \xi(p)}{d p}$, and formula (23), one can obtain

$$
\begin{gathered}
N\left(p_{0}\right)=\frac{1}{\pi} \int_{t_{0}}^{t_{x}} \ln \left[\frac{p(t)}{p_{0}}+\sqrt{\left(\frac{p(t)}{p_{0}}\right)^{2}-1}\right] \\
\frac{m a}{\sqrt{R_{2}^{2}-p(t)^{2}}} \frac{\mathrm{d} p_{\mathrm{s}}}{\mathrm{d} t} \mathrm{~d} t
\end{gathered}
$$

Note that Eq. (39) provides the Abel transform in the time interval $t_{0}, t_{x}$ where a layer contribution exists. The linear part of the regular trend due to the upper ionosphere is removed because of the second-order time derivative in Eq. (39). Therefore, Eq. (39) may give only that part of the refractivity altitude distribution which is connected with a sharp plasma layer contribution. A systematic error may be due to the impact of possible contributions in the upper ionosphere as it follows from the analytical model described in Sect. 3. The electron density distributions found by the inversion formula (39) are shown in Fig. 11.

The vertical gradients $\frac{d N_{\mathrm{e}}}{d h}\left[\frac{10^{3} \mathrm{el}}{\mathrm{cm}^{3} \mathrm{~km}}\right]$ and the electron content distributions $N_{\mathrm{e}}(h)\left[\frac{10^{3} \mathrm{el}}{\mathrm{cm}^{3}}\right]$ are shown in Fig. 11 (panels $\mathrm{a}, \mathrm{b}$, and c). Curves 1 and 2 correspond to the vertical gradient $\frac{d N_{\mathrm{e}}}{d h}$ and the altitude distribution of the electron content $N_{\mathrm{e}}$ retrieved from the RO eikonal data, respectively. The altitude $h$ of the RO ray perigee and the actual height $h^{\prime}$ of the layers are indicated on the top and bottom horizontal axes in Fig. 11 (panels a, b and c, respectively). The layers $\mathrm{a}$ and $\mathrm{b}$ are located on the ray part LT at approximate distances of $730 \mathrm{~km}$ and $620 \mathrm{~km}$ from the point T, respectively, with maximum gradients at the altitudes of $95 \mathrm{~km}$ and $117 \mathrm{~km}$, correspondingly (curves 1 and 2 in Fig. 11a, b). Layer c is located on the ray part GT at approximate distance of $620 \mathrm{~km}$ from the point $\mathrm{T}$ with a maximum vertical gradient at an altitude of $130 \mathrm{~km}$. According to Fig. 11, the variations of the vertical gradient of the electron density in layers a, b, and c are concentrated in the intervals $-\frac{2.8 \cdot 10^{5} \mathrm{el}}{\mathrm{cm}^{3} \mathrm{~km}}<\frac{d N(h)}{d h}<\frac{5.3 \cdot 10^{5} \mathrm{el}}{\mathrm{cm}^{3} \mathrm{~km}},-\frac{2.2 \cdot 10^{5} \mathrm{el}}{\mathrm{cm}^{3} \mathrm{~km}}<\frac{d N(h)}{d h}<\frac{7.8 \cdot 10^{5} \mathrm{el}}{\mathrm{cm}^{3} \mathrm{~km}}$ and $-\frac{2.0 \cdot 10^{5} \mathrm{el}}{\mathrm{cm}^{3} \mathrm{~km}}<\frac{d N(h)}{d h}<\frac{3.2 \cdot 10^{5} \mathrm{el}}{\mathrm{cm}^{3} \mathrm{~km}}$, respectively. These magnitudes are typical for the intense sporadic E-layers (Kelley and Heelis, 2009). The altitude interval of the layers amplitude response is nearly equal to the height interval of the variations in the electron density and its gradient. The maximum values of the electron content in sporadic E-layers are usually attributed to the impact of the wind shears (Kelley and Heelis, 2009). The gradient of the electron content can correspond to the direction of different kinds of wave fronts influencing the ionospheric plasma distribution. Therefore, the RO method is capable of locating the wind shear in the lower ionosphere, and, in the case of internal gravity waves 
(GWs), determining the inclination of the wave vector to the vertical direction. This can be used to find the angular frequency of GWs (Gubenko et al., 2008). The innovative technique proposed can extend the application domain of the RO method. Additional validation of this method through comparison with ground-based ionosonde information is the task for the future work.

\section{Conclusions}

An analytical model is introduced that accounts for the local mechanism of multiple-RO ionospheric phenomena and involves horizontal gradients in the ionosphere. The model gives analytical expressions for the excess phase path, bending angle, and refractive attenuation of electromagnetic waves propagating through the disturbed ionosphere. As applied to the RO data analysis, the analytical model associates the Coherent variations of the eikonal and intensity of the RO signals at $30-90 \mathrm{~km}$ altitude of the RO ray perigee to the contribution of inclined layers in the ionosphere. This allows one to eliminate systematic errors in the RO estimation of the altitude of ionospheric layers. The analytical model makes it possible to apply the Abel transform to estimating the electron density distribution in the inclined ionospheric plasma layers. The quality of CHAMP RO data and the analytical model have demonstrated the important application of a comparative analysis of the amplitude and phase channels of satellite radio-holograms to classifying the ionospheric influence on the RO signals. The amplitude variations of the RO signal are similar to the noisy (C-type) and regular (S-type) scintillations detected earlier in the satellite-to-Earth communication link. These types correspond to different phenomena that accompany radio wave propagation through the ionosphere. The regular types correspond to wave-like structures and layers. The noisy events may be due to the scattering by irregularities and turbulence in the ionosphere. These types are useful for understanding the nature of the ionospheric interferences in the satellite-to-satellite and satelliteto-Earth communication links.

A necessary and sufficient criterion is obtained for a layer to be located at the $\mathrm{RO}$ ray perigee. This criterion gives both qualitative and quantitative estimation of the displacement of an ionospheric and/or atmospheric layer from the RO ray perigee. This is important, in particular, for determining the location of wind shears and directions of the internal wave propagation in the lower ionosphere, and, possibly, in the atmosphere. The innovative technique proposed can extend the application domain of the RO method. Additional validation of this method through comparison with ground-based ionosonde information is the task for the future work.
Acknowledgements. We are grateful to GFZ-Potsdam for access to the CHAMP RO data. This research is partially supported through an Australian Research Council project (ARC- LP0883288), the Australia Space Research Program platform technology project and International Science Linkage project (DIISR/ISL-CG130127) from the Department of Industry, Innovation, Science and Research endorsed to research consortiums led by RMIT University. Work has been partly supported by National Science Council and National Space Organization of Taiwan, R.O.C., grants NSC 982111-M-008-012-MY3 and 98-NSPO(B)-IC-FA07-01(X), by grant no. 10-02-01015-a, Russian Foundation of Basic Research, and by program OFN-15 and OFN-6 of Russian Academy of Sciences.

Edited by: K. B. Lauritsen

\section{References}

Ahmad, B. and Tyler, L.: Systematic errors in atmospheric profiles obtained from Abelian inversion of radio occultation data: Effects of large-scale horizontal gradients, J. Geophys. Res., 104, 3971-3992, 1999.

Gorbunov, M. E.: Ionospheric correction and statistical optimization of radio occultation data, Radio Sci., 37, 171-179, 2002.

Gorbunov, M. E. and Kirchengast, G.: Processing X/K Band Radio Occultation Data in Pres-ence of Turbulence, Radio Sci., 40, RS6001, doi:10.1029/2005RS003263, 2005.

Gorbunov, M. E. and Lauritsen, K. B.: Analysis of wave fields by Fourier integral operators and its application for radio occultations, Radio Sci., 39, RS4010, doi:10.1029/2003RS002971, 2004.

Gorbunov, M. E., Gurvich, A. S., and Shmakov, A. V.: Backpropagation and radio-holographic methods for investigation of sporadic ionospheric E-layers from Microlab-1 data, Int. J. Remote Sens., 23, 675-685, 2002.

Gorbunov, M. E., Lauritsen, K. B., and Leroy, S. S.: Application of Wigner distribution function for analysis of radio occultations, Radio Sci., 45, RS6011, doi:10.1029/2010RS004388, 2010.

Gubenko, V. N., Pavelyev, A. G., and Andreev, V. E.: Identification of wave origin of temperature fluctuations and determination of the intrinsic frequency of internal gravity waves in the Earth's stratosphere derived from radio occultation data J. Geophys. Res., 113, D08109, doi:10.1029/2007JD008920, 2008.

Hajj, G. A. and Romans, L. J.: Ionospheric electron density profiles obtained with the Global Positioning System: Results from GPS/MET experiment, Radio Sci., 33, 175-190, 1998.

Hinson, D. P., Flasar, F. M., Schinder, A., Twicken, J. D., and Herrera, R. G.: Jupiter's Ionosphere: Results from the first Galileo radio occultation experiment, Geophys. Res. Lett., 24, 2107, doi:10.1029/97GL01608, 1997.

Hocke, K.: Inversion of GPS meteorology data, Ann. Geophysicae, 15, 143-152, 1997.

Hocke, K., Pavelyev, A., Yakovlev, O., Barthes, L., and Jakowski, N.: RO data analysis by radio holographic method, JASTP, 61, 1169-1177, 1999.

Igarashi, K., Pavelyev, A. G., Hocke, K., Kucherjavenkov, A. I., Matugov, S. S., Yakovlev O. I., Pavelyev, D., and Zakharov, A.: Radio holographic principle for observing natural processes in 
the atmosphere and retrieving meteorological parameters from radio occultation data, Earth, Planet. Space, 52, 868-875, 2000.

Igarashi, K., Pavelyev, A. G., Hocke, K., Pavelyev, D., and Wickert, J.: Observation of wave structures in the upper atmosphere by means of radio holographic analysis of the RO data, Adv. Space Res., 27, 1321-1327, 2001.

Jensen, A. S., Lohmann, M., Benzon, H.-H., and Nielsen, A. S.: Full spectrum inversion of radio occultation signals, Radio Sci., 38, 1040, doi:10.1029/2002RS002763, 2003.

Jakowski, N., Leitinger, R., and Angling, M.: Radio occultation techniques for probing the ionosphere, Ann. Geophys., supplement to 47, 1049-1066, 2004.

Karasawa, Y., Yasukawa, K., and Yamada, M.: Ionospheric scintillation measurement at $1.5 \mathrm{GHz}$ in mid-latitude region, Radio Sci., 20, 643-651, 1985.

Kelley, M. C. and Heelis, R. A.: The earth's ionosphere: plasma physics and electrodynamics. Elsevier Science, 556 pp. Volume 96 International Geophysics Second Edition Cornell University Engineering School of Electrical Engineering Academic Press, ISBN 10: 0120884259/0-12-088425-9, ISBN 13: 9780120884254, 2009.

Kunitsyn, V. E. and Tereshchenko, E. D.: Ionospheric Tomography Springer-Verlag, Berlin, 2003.

Kursinski, E. R., Hajj, G. A., Schofield, J. T., and Linfield, R. P.: Observing Earth's atmosphere with radio occultation measurements using the global positioning system, J. Geophys. Res., 102, 23429-23465, 1997.

Liou, Y. A. and Pavelyev, A. G.: Simultaneous observations of radio wave phase and intensity variations for locating the plasma layers in the ionosphere, Geophys. Res. Lett., 33, L23102, doi:10.1029/2006GL027112, 2006.

Liou, Y.-A., Pavelyev, A. G., Huang, C.-Y., Igarashi, K., and Hocke, K.: Simultaneous observation of the vertical gradients of refractivity in the atmosphere and electron density in the lower ionosphere by radio occultation amplitude method, Geophys. Res. Lett., 29, 431-434, 2002.

Liou, Y.-A., Pavelyev, A. G., Huang, C.-Y., Igarashi, K., Hocke, K., and Yan, S. K.: Analytic method for observation of the GW using RO data, Geophys. Res. Lett., 30, ASC, 11-15, 2003.

Liou, Y.-A., Pavelyev, A. G., Pavelyev, A. A., Wickert, J., and Schmidt, T.: Analysis of atmospheric and ionospheric structures using the GPS/MET and CHAMP radio occultation data base: A methodological review, GPS Solutions, 9, 122-143, 2005.

Liou, Y.-A., Pavelyev, A. G., Liu, S.-F., Pavelyev, A. A., Yen, N., Huang, C.-Y., and Fong, C.-J.: FORMOSAT-3/COSMIC GPS Radio Occultation Mission: Preliminary Results, IEEE T. Geosci. Remote Sens., 45, 3813-3826, November 2007.

Liou, Y.-A., Pavelyev, A. G., Matyugov, S. S., Yakovlev, O. I., and Wickert, J.: Radio Occultation Method for Remote Sensing of the Atmosphere and Ionosphere, edited by: Liou, Y. A., INTECH Published by In-The Olajnica 19/2, 32000 Vukovar, Croatia, 170 pp. 45 ill., ISBN 978-953-7619-60-2, 2010.

Lindal, G. F., Lyons, J. R., Sweetnam, D. N., Eshleman, V. R., Hinson, D. P., and Tyler, G. L.: The atmosphere of Uranus: Results of radio occultation measurements with Voyager, J. Geophys. Res., 92, 14987-15001, 1987.

Marouf, E. A. and Tyler, G. L.: Microwave edge diffraction by features in Saturn's rings: Observations with Voyager 1, Science, 217, 243-245, 1982.
Melbourne, W. G.: Radio Occultations Using Earth Satellites: A Wave Theory Treatment, Jet Propulsion Laboratory California Institute of Technology, Monograph 6, Deep space communications and navigation series, Issued by the Deep Space Communications and Navigation Systems Center of Excellence Jet Propulsion Laboratory California Institute of Technology, edited by: Yuen, J. H., 610 pp., 2004.

Melbourne, W. G., Davis, E. S., Duncan, C. B., Hajj, G. A., Hardy, K. R., Kursinski, E. R., Meehan, T. K., Young, L. E., and Yunck, T. P.: The Application of Spaceborne GPS to Atmospheric Limb Sounding and Global Change Monitoring, JPL Publication, 9418, 147 pp., 1994.

Pavelyev, A. G. and Kucherjavenkov, A. I.: Refractive attenuation in the planetary atmospheres, Radio Eng. Electron. Phys., 23, 13-19, 1978.

Pavelyev, A. G., Liou, Y.-A., Huang, C. Y., Reigber, C., Wickert, J., Igarashi, K., and Hocke, K.: Radio holographic method for the study of the ionosphere, atmosphere and terrestrial surface using GPS occultation signals, GPS Solutions, 6, 101-108, 2002.

Pavelyev, A. G., Liou, Y. A., and Wickert, J.: Diffractive vector and scalar integrals for bistatic radio- holographic remote sensing, Radio Sci., 39, RS4011, doi:10.1029/2003RS002935, 2004.

Pavelyev, A. G., Liou, Y. A., Wickert, J., Schmidt, T., Pavelyev, A. A., and Liu, S. F.: Effects of the ionosphere and solar activity on radio occultation signals: Application to CHAllenging Minisatellite Payload satellite observations, J. Geophys. Res., 112, A06326, doi:10.1029/2006JA011625, 2007.

Pavelyev, A. G., Wickert, J., and Liou, Y.-A.: Localization of plasma layers in the ionosphere based on observing variations in the amplitude and phase of radiowaves along the satellite-tosatellite path, Radiophys. Quantum El., 51, 1-8, 2008 a.

Pavelyev, A. G., Liou, Y.-A., Wickert, J., Pavelyev, A. A., Schmidt, T., Igarashi, K., and Matyugov, S. S.: Location of layered structures in the ionosphere and atmosphere by use of GPS occultation data, Adv. Space Res., 42, 224-228, 2008b.

Pavelyev, A. G., Liou, Y. A., Wickert, J., Gavrik, A. L., and Lee, C. C.: Eikonal acceleration technique for studying of the Earth and planetary atmospheres by radio occultation method, Geophys. Res. Lett., 36, L21807, doi:10.1029/2009GL040979, 2009.

Pavelyev, A. G., Liou, Y.-A., Wickert, J., Schmidt, T., Pavelyev, A. A., and Matyugov, S. S.: Phase acceleration: a new important parameter in GPS occultation technology, GPS Solutions, 14, 3 14, doi:10.1007/s10291-009-0128-1, 2010a.

Pavelyev, A. G., Liou, Y. A., Wickert, J., Zhang, K., Wang, C.-S., and Kuleshov, Yu.: Analytical model of electromagnetic waves propagation and location of inclined plasma layers using occultation data. Progress in Electromagnetics Research (PIER), 2010, 106, 177-202, doi:10.2528/PIER10042707, 2010b.

Sokolovskiy, S. V.: Inversion of RO amplitude data, Radio Sci., 35, 97-105, 2000

Sokolovskiy, S. V., Schreiner, W., Rocken, C., and Hunt, D.: Detection of high-altitude ionospheric irregularities with GPS/MET, Geophys. Res. Lett., 29, 621-625, 2002.

Steiner, A. K. and Kirchengast, G.: GW spectra from GPS/MET occultation observations, J. Atmos. Ocean. Tech., 17, 495-503, 2000.

Steiner, A. K., Kirchengast, G., and Landreiter, H. P.: Inversion, error analysis, and validation of GPS/MET occultation data, Ann. Geophys., 17, 122-138, 1999, 
http://www.ann-geophys.net/17/122/1999/.

Vorob'ev, V. V. and Krasilnikova, T. G.: Estimation of accuracy of the atmosphere refractive index recovery from Doppler shift measurements at frequencies used in the NAVSTAR system, Izv. Russ. Acad. Sci., Physics of the Atmosphere and Ocean, Engl. Transl., 29, 602-609, 1994.

Vorob'ev, V. V., Gurvich, A. S., Kan, V., Sokolovskiy, S. V., Fedorova, O. V., and Shmakov, A. V.: The structure of the ionosphere from the GPS-"Microlab-1" radio occultation data: Preliminary results, Cosmic Res., 4, 74-83, 1997 (in Russian).

Ware, R., Exner, M., Feng, D., Gorbunov, M., Hardy, K., Herman, B., Kuo, Y.-H., Meehan, T., Melbourn, W., Rocken, C., Schreiner, W., Sokolovskiy, S., Solheim, F., Zou, X., Anthes, R., Businger, S., and Trenberth, K.: GPS soundings of the atmosphere from low earth orbit: Preliminary results, B. Am. Meteorol. Soc., 77, 19-40, 1996.
Wickert, J., Reigber, C., Beyerle, G., Konig, R., Marquardt, C., Schmidt, T., Grunwaldt, L., Galas, R., Meehan, T. K., Melbourne, W. G., and Hocke, K.: Atmosphere sounding by GPS radio occultation: First results from CHAMP, Geophys. Res. Lett., 28, 3263-3266, 2001.

Wickert, J., Pavelyev, A. G., Liou, Y. A., Schmidt, T., Reigber, Ch., Igarashi, K., Pavelyev, A. A., and Matyugov, S.: Amplitude scintillations in GPS signals as a possible indicator of ionospheric structures, Geophys. Res. Lett., 31, L24801, doi:10.1029/2004GL020607, 2004.

Yakovlev, O. I.: Space Radio Science, 306 pp., Taylor and Francis, London, 2003. 